\title{
Fixed-order Decentralized/Distributed Control of Islanded Inverter-interfaced Microgrids
}

\author{
Mahdieh S. Sadabadi ${ }^{\mathrm{a}}$, Alireza Karimi ${ }^{\mathrm{a}}$, Houshang Karimi ${ }^{\mathrm{b}}$ \\ mahdieh.sadabadi@epfl.ch, alireza.karimi@epfl.ch,houshang.karimi@polymtl.ca \\ ${ }^{a}$ Automatic Control Laboratory, Ecole Polytechnique Fédérale de Lausanne (EPFL), \\ Lausanne, Switzerland \\ ${ }^{b}$ Department of Electrical Engineering, Ecole Polytechnique de Montréal, Montréal, \\ Canada
}

\begin{abstract}
This paper focuses on the problem of voltage control of islanded inverterinterfaced microgrids consisting of several distributed generation (DG) units with parallel structure. The main objectives are to (i) design a decentralized/distributed voltage controller with minimum information exchange between DG units and their local controllers (ii) design a fixed-/low-order dynamic output feedback controller which ensures stability as well as desired performance of the microgrid system in spite of load parameter uncertainties. To this end, the problem is formulated as an optimization problem which is the minimization of the cardinality of a pattern matrix subject to an $H_{\infty}$ performance constraint. Since the problem is intrinsically non-convex, a convex design procedure for the controller synthesis is proposed in this paper. The effectiveness of the proposed controller is evaluated through simulation studies and Hardware-In-the-Loop (HIL) verifications. The simulation and experimental results demonstrate that the effectiveness of the proposed control strategy.
\end{abstract}

Keywords: $H_{\infty}$ control, Distributed/decentralized control, Control structure design, Fixed-order controller, Islanded inverter-interfaced microgrids, Linear Matrix Inequality (LMI)

\section{INTRODUCTION}

Nowadays the growth of electricity demand, the critical shortages of fossil fuels, and global warming caused by greenhouse-gas-effect have negatively 
impacted on conventional power systems. The problems have been tackled alternatively through an efficacious integration and coordination of distributed generation (DG) units such as photovoltaics (PV), wind power, etc.

Reliable integration of DG units into power systems can be achieved by means of microgrids which are small electrical networks heterogeneously composed of DG units, loads, and energy storage systems [1]. Renewable energy sources are normally interfaced to the microgrid through power electronic converters acting as voltage sources [2].

Microgrids normally operate in grid-connected mode where they are connected to the main grid at Point of Common Coupling (PCC). Under this connection scheme, the microgrid voltage and frequency are predominantly determined by the main grid while the microgrid control system accurately shares active and reactive power among DG units and controls the power exchange between the microgrid and the main grid. Due to intentional (scheduled)/unintentional reasons, the microgrids can experience islanding conditions where they are disconnected from the main grid [3]. In this case, due to the power mismatch between the DGs and the loads, voltage and frequency of the loads deviate from their rated values and the islanded microgrid eventually becomes unstable. This operation mode of microgrids is more challenging than the grid-connected mode because accurate load sharing mechanisms are required to balance the power mismatch [1]. Therefore, upon the islanding condition, a new microgrid control strategy must come into service in order to provide voltage and frequency stability as well as a proper power sharing among DG units [4].

A control strategy ubiquitously used for the control of microgrids is droop control which relies on the principle of power balance of a classical synchronous generator in conventional power networks (see, e.g., [5, 6, 7, 8, 9, $10,2,11,12,13,14])$. In the power systems based on rotating generators, frequency (rotor speed) is dependent on active power balance, i.e. the frequency is dropped when the injected active power increases [15]. The idea of the so-called "droop" controllers has been developed by Chandorkar et al [16]. From a control point of view, droop control is a decentralized proportional controller maintaining the voltage and frequency stability of the microgrids [17]. The main advantage of droop-based control is the elimination of the communication links among DG local controllers. Nonetheless, the droop-based approaches suffer from several drawbacks including (i) poor transient performance, (ii) poor performance for mixed-line microgrids with resistive-inductive line conditions, and (iii) coupled dynamics between active 
and reactive power.

In addition to the droop-based control strategies, non-droop-based approaches for voltage and frequency control of the islanded microgrids have also been developed, e.g. [18, 19, 20, 21, 22, 23, 24, 25, 26]. The proposed methods regulate the voltage of single-DG-unit [19, 20, 21, 24, 25] and/or multi-DG-unit microgrids $[23,26]$. In these methods, the frequency of each DG unit is controlled through an internal oscillator in the open-loop manner with $\omega_{0}=2 \pi f_{0}$, where $f_{0}$ is the nominal system frequency. All oscillators are synchronized by a common time reference signal according to a global positioning system (GPS) [26].

Although extensive research has been carried out on the development of non-droop-based control of micorgrids, they suffer from one or more of the following drawbacks: (i) inability to guarantee robust stability and/or robust performance with respect to load parameter variations [24, 20, 23]. (ii) inapplicability to multiple-DG microgrids, e.g. [24, 20], (iii) high-order controllers, e.g. [21, 25], and (iv) centralized control strategy, e.g. [18].

To overcome the disadvantages of the existing microgrid control approaches, various challenges associated with robustness to load parametric uncertainties, design of decentralized/distributed controllers with minimum communication links among DGs, and low-complexity of the local controllers must be addressed. Therefore, the problem of microgrid control is translated into fixed-/low-order decentalized/distributed controller design of interconnected systems with parameter uncertainties.

In the distributed control strategy, there exist several communication links between the local controllers and the subsystems according to the control structure. Most of available distributed control approaches assume that the control structure is given a priori [27]. However, it is possible that the assumed control structure is not the best one which can be taken into consideration. Moreover, it is generally difficult to select the structure of the controller in advance. Therefore, the question arises is that in an interconnected system, what is the best control structure, in terms of the connections between the local controllers and the subsystems, to satisfy the given control objectives?

This question has been recently addressed by some researchers in [28, 29, $30,31,32]$. They have focused on the problem of sparse static output/state feedback controller design where the gain between the subsystems' inputs and outputs/states is sparsified [28, 29, 30, 31]. In this way, the amount of information exchange between subsystems and the controller is reduced. The 
results of [28] is extended to sparse dynamic output control design in [32]. However, in [32] some parts of the controller structure, i.e. the structure of matrices $A_{c}, B_{c}$, and $C_{c}$, have been specified a priori. In all these approaches, the sparsity is formulated in terms of the cardinality of the gain matrix defined as the number of its non-zero elements. The communication links between the local controllers has not been considered in these approaches. Moreover, the robustness of the controller to system parameter uncertainties is another important issue which has not been taken into consideration. In an uncertain interconnected system, the control structure and the control parameters have to be designed for a prescribed family of models.

In this paper, a new LMI-based approach to the problem of fixed-order decentralized/distributed $H_{\infty}$ control of interconnected systems subject to polytopic uncertainty is proposed. In this approach, the controller structure as well as the controller parameters are simultaneously designed. The objectives are achieved by transforming the problem into a non-convex optimization problem in which the number of non-zero elements (cardinality) of a pattern matrix is minimized subject to an $H_{\infty}$ performance constraint. The non-convex cardinality of the pattern matrix is relaxed by a weighted $\ell_{1}$ norm [33] and an inner convex approximation of fixed-order $H_{\infty}$ controllers for polytopic systems is then given. Therefore, the problem of fixed-order decentralized/distributed $H_{\infty}$ controller design can be solved by finding a feasible solution of an iterative convex optimization problem. Then, the proposed robust fixed-order decentalized/distributed control strategy is applied to an LTI model of a microgrid in a rotating reference frame ( $d q$-reference frame). The designed controller is able to overcome the limitations of the existing droop-based controllers which are only appropriate for microgrids with dominantly inductive and/or resistive power lines. Furthermore, opposed to most non-droop-based control methods, the controller guarantee the robust stability and robust performance against the load parameter changes. Simulation studies in MATLAB and experimental results using real-time hardware-inthe-loop (HIL) environment demonstrate the effectiveness of the designed controllers.

The organization of the paper is as follows. The problem formulation is presented in Section 2. A convex solution to the problem of fixed-order decentralized/distributed control of polytopic systems with guaranteed $H_{\infty}$ performance is provided in Section 3. Section 4 is devoted to the design of robust fixed-order voltage controllers for islanded inverter-interfaced microgrids. Section 5 concludes the paper. 
Throughout the paper, matrices $I$ and 0 are the identity matrix and the zero matrix of appropriate dimensions, respectively. The symbols $T$ and $\star$ denote the matrix transpose and symmetric blocks, respectively. For symmetric matrices, $P>0(P<0)$ indicates the positive-definiteness (negativedefiniteness).

\section{PROBLEM FORMULATION}

\subsection{System model}

Consider a linear time-invariant interconnected system consisting of $N$ subsystems described by the following state space equations:

$$
\begin{aligned}
\dot{x}_{g_{i}}(t) & =A_{g_{i i}} x_{g_{i}}(t)+\sum_{j=1(j \neq i)}^{N} A_{g_{i j}} x_{g_{j}}(t)+\sum_{j=1}^{N} B_{w_{i j}} w_{j}(t)+B_{g_{i}} u_{i}(t) \\
z_{i}(t) & =\sum_{j=1}^{N} C_{z_{i j}} x_{g_{j}}(t)+\sum_{j=1}^{N} D_{z w_{i j}} w_{j}(t)+D_{z u_{i}} u_{i}(t) \\
y_{i}(t) & =C_{g_{i}} x_{g_{i}}(t)+\sum_{j=1}^{N} D_{w_{i j}} w_{j}(t)
\end{aligned}
$$

where $x_{g_{i}} \in \mathbb{R}^{n}, u_{i} \in \mathbb{R}^{n_{i}}, w_{i} \in \mathbb{R}^{r}, y_{i} \in \mathbb{R}^{n_{o}}$, and $z_{i} \in \mathbb{R}^{s}$ are the state, the control input, the exogenous input, the measured output, and the controlled output of the $i^{\text {th }}$ subsystem, respectively. The state space matrices $A_{g_{i i}}, A_{g_{i j}}$, $B_{g_{i}}, C_{g_{i}}, B_{w_{i j}}, C_{z_{i j}}, D_{z u_{i}}, D_{z w_{i j}}$, and $D_{w_{i j}}$ are of appropriate dimensions. Matrix $A_{g_{i j}}=0$ if and only if there is no interaction between the subsystems $i$ and $j$. It is assumed that the state space matrices either $\left(A_{g_{i i}}, A_{g_{i j}}, B_{g_{i}}\right)$ or $\left(A_{g_{i i}}, A_{g_{i j}}, C_{g_{i}}\right)$ belong to a polytopic uncertainty region as follows:

$$
\left(A_{g_{i i}}(\lambda), A_{g_{i j}}(\lambda), B_{g_{i}}(\lambda), C_{g_{i}}(\lambda)\right)=\sum_{l=1}^{q} \lambda_{l}\left(A_{g_{i i}}^{l}, A_{g_{i j}}^{l}, B_{g_{i}}^{l}, C_{g_{i}}^{l}\right)
$$

for $i, j=1, \ldots, N$, where $\lambda=\left[\lambda_{1} \cdots \lambda_{q}\right]^{T}$ is in the following unit simplex $\Lambda$ :

$$
\Lambda=\left\{\lambda \mid \sum_{l=1}^{q} \lambda_{l}=1, \quad \lambda_{l} \geq 0 ; \quad l=1, \ldots, q\right\}
$$

where $q$ is the number of vertices of the polytopic system and matrices $A_{g_{i i}}^{l}$, $A_{g_{i j}}^{l}, B_{g_{i}}^{l}$, and $C_{g_{i}}^{l}$ are the $l^{\text {th }}$ vertex of the polytope. In what follows, we assume that $\left(A_{g_{i i}}, A_{g_{i j}}, B_{g_{i}}\right)$ belongs to the polytopic uncertainty domain. 
The whole network of $N$ subsystems is presented by the following equations:

$$
\begin{aligned}
\dot{x}_{g}(t) & =A_{g}(\lambda) x_{g}(t)+B_{g}(\lambda) u(t)+B_{w} w(t) \\
z(t) & =C_{z} x_{g}(t)+D_{z u} u(t)+D_{z w} w(t) \\
y(t) & =C_{g} x_{g}(t)+D_{w} w(t)
\end{aligned}
$$

where

$$
\begin{aligned}
x_{g}(t) & =\left[x_{g_{1}}^{T}(t), \ldots, x_{g_{N}}^{T}(t)\right]^{T}, & u(t)=\left[u_{1}^{T}(t), \ldots, u_{N}^{T}(t)\right]^{T} \\
w(t) & =\left[w_{1}^{T}(t), \ldots, w_{N}^{T}(t)\right]^{T}, & z(t)=\left[z_{1}^{T}(t), \ldots, z_{N}^{T}(t)\right]^{T} \\
y(t) & =\left[y_{1}^{T}(t), \ldots, y_{N}^{T}(t)\right]^{T} &
\end{aligned}
$$

and the state space matrices are given as follows:

$$
\begin{aligned}
& A_{g}(\lambda)=\left[\begin{array}{c:c:c}
A_{g_{11}}(\lambda) & \ldots & A_{g_{1 N}}(\lambda) \\
\hdashline \vdots & \ddots & \vdots \\
\hdashline A_{g_{N 1}}(\lambda) & \cdots & A_{g_{N N}}(\lambda)
\end{array}\right], \quad B_{w}=\left[\begin{array}{c:c:c}
B_{w_{11}} & \ldots & B_{w_{1 N}} \\
\hdashline \vdots & \ddots & \vdots \\
\hdashline B_{w_{N 1}} & \ldots & B_{w_{N N}}
\end{array}\right] \\
& C_{z}=\left[\begin{array}{c:c:c}
C_{z_{11}} & \ldots & C_{z_{1 N}} \\
\hdashline \vdots & \ddots & \vdots \\
\hdashline C_{z_{N 1}} & \ldots & C_{z_{N N}}
\end{array}\right], \quad D_{z w}=\left[\begin{array}{c:c:c}
D_{z w_{11}} & \ldots & D_{z w_{1 N}} \\
\hdashline \vdots & \ddots & \vdots \\
\hdashline D_{z w_{N 1}} & \ldots & D_{z w_{N N}}
\end{array}\right], \\
& D_{w}=\left[\begin{array}{c:c:c}
D_{w_{11}} & \cdots & D_{w_{1 N}} \\
\hdashline \vdots & \ddots & \vdots \\
\hdashline D_{w_{N 1}} & \cdots & D_{w_{N N}}
\end{array}\right]
\end{aligned}
$$

and

$$
\begin{aligned}
B_{g}(\lambda) & =\operatorname{diag}\left(B_{g_{1}}(\lambda), \ldots, B_{g_{N}}(\lambda)\right) \\
C_{g} & =\operatorname{diag}\left(C_{g_{1}}, \ldots, C_{g_{N}}\right) \\
D_{z u} & =\operatorname{diag}\left(D_{z u_{1}}, \ldots, D_{z u_{N}}\right)
\end{aligned}
$$

\subsection{Dynamic output feedback controller}

It is assumed that there is one local controller corresponding to each subsystem described by:

$$
\begin{aligned}
\dot{x}_{c_{i}}(t) & =\sum_{j=1}^{N} A_{c_{i j}} x_{c_{j}}(t)+\sum_{j=1}^{N} B_{c_{i j}} y_{j}(t) \\
u_{i}(t) & =\sum_{j=1}^{N} C_{c_{i j}} x_{c_{j}}(t)+\sum_{j=1}^{N} D_{c_{i j}} y_{j}(t)
\end{aligned}
$$


for $i=1, \ldots, N$, where $x_{c_{i}} \in \mathbb{R}^{m}$ is the state vector of the $i^{t h}$ local controller. The controller matrices $A_{c_{i j}}, B_{c_{i j}}, C_{c_{i j}}$, and $D_{c_{i j}}$ are of appropriate dimensions. According to this structure, each local controller uses the outputs of its own subsystem and other subsystems as well as the states of other local controllers. The centralized controller $K$ with this structure is given by:

$$
\begin{aligned}
\dot{x}_{c}(t) & =A_{c} x_{c}(t)+B_{c} y(t) \\
u(t) & =C_{c} x_{c}(t)+D_{c} y(t)
\end{aligned}
$$

where

$$
\begin{aligned}
& x_{c}(t)=\left[x_{c_{1}}^{T}(t), \ldots, x_{c_{N}}^{T}(t)\right]^{T} \\
& A_{c}=\left[\begin{array}{c:c:c}
A_{c_{11}} & \ldots & A_{c_{1 N}} \\
\hdashline \vdots & \ddots & \vdots \\
\hdashline A_{c_{N 1}} & \cdots & A_{c_{N N}}
\end{array}\right], \quad B_{c}=\left[\begin{array}{c:c:c}
B_{c_{11}} & \ldots & B_{c_{1 N}} \\
\hdashline \vdots & \ddots & \vdots \\
\hdashline B_{c_{N 1}} & \cdots & B_{c_{N N}}
\end{array}\right] \\
& C_{c}=\left[\begin{array}{c:c:c}
C_{c_{11}} & \ldots & C_{c_{1 N}} \\
\hdashline \vdots & \ddots & \vdots \\
\hdashline C_{c_{N 1}} & \cdots & C_{c_{N N}}
\end{array}\right], \quad D_{c}=\left[\begin{array}{c:c:c}
D_{c_{11}} & \ldots & D_{c_{1 N}} \\
\hdashline \vdots & \ddots & \vdots \\
\hdashline D_{c_{N 1}} & \ldots & D_{c_{N N}}
\end{array}\right]
\end{aligned}
$$

\subsection{Control Structure Selection}

To select an appropriate control structure, it is important to determine whether there exists any link between the local controller $i$ and the subsystem $j$ as well as the local controller $j$. In other words, the outputs of the subsystem $j$ do not contribute to the construction of the control inputs $i$ if and only if both $B_{c_{i j}}=0$ and $D_{c_{i j}}=0$. Moreover, there is no communication link between the local controllers $i$ and $j$ if and only if both $A_{c_{i j}}=0$ and $C_{c_{i j}}=0$.

The main objective is to design a controller such that each local controller uses a minimum amount of information exchange between the subsystems and the local controllers. In order to design such controller, the following pattern matrix $\mathcal{Z}(K)=\left[z_{i j}\right]$ is defined:

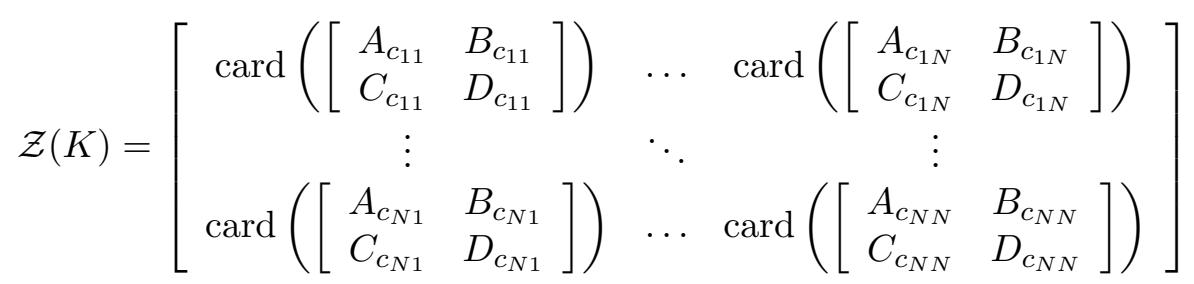

where $\operatorname{card}(\cdot)$ is the cardinality operator defined as the number of non-zero elements of $(\cdot)$. Element $z_{i j}$ of $\mathcal{Z}(K)$ represents the communication links 
between the local controller $i$ and the subsystem and the local controller $j$. The number of the non-zero elements of $\mathcal{Z}(K)$ is defined as the number of the communication links of the controller. Note that $z_{i j}=0$ if and only if $\left[\begin{array}{cc}A_{c_{i j}} & B_{c_{i j}} \\ C_{c_{i j}} & D_{c_{i j}}\end{array}\right]=0$, i.e. there exists no link between the local controller $i$ and the subsystem $j$ with its corresponding controller. The control structure is represented by a binary information flow matrix $\mathcal{I}(K)=\left[I_{i j}\right]$ determined as follows:

$$
I_{i j}=\operatorname{sgn}\left(z_{i j}\right)
$$

where sgn is the signum function. When all entries of matrix $\mathcal{I}(K)$ are equal to 1 , the corresponding controller is centralized and when $\mathcal{I}(K)$ is diagonal, the corresponding controller is fully decentralized.

To find a controller structure with minimum communication links between the subsystems and the local controllers, matrix $\mathcal{Z}(K)$ should be as sparse as possible. The sparsity of this matrix can be presented by its cardinality which is equal to the number of its non-zero elements.

\subsection{Closed-loop system}

The state space representation of the closed-loop system $H_{z w}(\lambda)$, transfer function matrix from $w$ to $z$, can be written as:

$$
\begin{aligned}
\dot{x}(t) & =A(\lambda) x(t)+B(\lambda) w(t) \\
z(t) & =C(\lambda) x(t)+D(\lambda) w(t)
\end{aligned}
$$

where $x(t)=\left[\begin{array}{ll}x_{g}^{T}(t) & x_{c}^{T}(t)\end{array}\right]^{T}$ and

$$
\begin{aligned}
A(\lambda) & =\left[\begin{array}{cc}
A_{g}(\lambda)+B_{g}(\lambda) D_{c} C_{g} & B_{g}(\lambda) C_{c} \\
B_{c} C_{g} & A_{c}
\end{array}\right], \quad B(\lambda)=\left[\begin{array}{c}
B_{w}+B_{g}(\lambda) D_{c} D_{w} \\
B_{c} D_{w}
\end{array}\right] \\
C(\lambda) & =\left[\begin{array}{ll}
C_{z}+D_{z u} D_{c} C_{g} & D_{z u} C_{c}
\end{array}\right], \quad D(\lambda)=D_{z w}+D_{z u} D_{c} D_{w}
\end{aligned}
$$

The closed-loop state matrix $A(\lambda)$ is called robustly stable if all its eigenvalues have strictly negative real part for all $\lambda \in \Lambda$.

The transfer function of the vertices of the closed-loop polytopic system $H_{z w}(\lambda)$ are given as follows:

$$
H_{z w}^{l}(s)=\left[\begin{array}{c|c}
A^{l} & B^{l} \\
\hline C^{l} & D^{l}
\end{array}\right] ; \quad l=1, \ldots, q
$$


where

$$
\begin{aligned}
& A^{l}=\left[\begin{array}{cc}
A_{g}^{l}+B_{g}^{l} D_{c} C_{g} & B_{g}^{l} C_{c} \\
B_{c} C_{g} & A_{c}
\end{array}\right], \quad B^{l}=\left[\begin{array}{c}
B_{w}+B_{g}^{l} D_{c} D_{w} \\
B_{c} D_{w}
\end{array}\right] \\
& C^{l}=\left[\begin{array}{ll}
C_{z}+D_{z u} D_{c} C_{g} & D_{z u} C_{c}
\end{array}\right], \quad D^{l}=D_{z w}+D_{z u} D_{c} D_{w}
\end{aligned}
$$

\section{LMI REPRESENTATION OF FIXED-ORDER SPARSE $\boldsymbol{H}_{\infty}$ CONTROLLER DESIGN}

The problem of fixed-order decentralized/distributed control of LTI interconnected systems subject to polytopic-type uncertainty in (1)-(2) can be summarized as:

Given a linear dynamical interconnected system consists of $N$ subsystems with polytopic uncertainty, design a fixed-order dynamic output feedback controller $K$ such that

1. The cardinality of $\mathcal{Z}(K)$ is minimized (Control Structure Selection).

2. Closed-loop system is robustly stable and the performance criterion $\left\|H_{z w}(\lambda)\right\|_{\infty}^{2}<\mu$ is guaranteed (Controller Design).

The aforementioned conditions can be formulated as the following optimization problem:

$$
\begin{array}{ll}
\min _{K} & \operatorname{card}(\mathcal{Z}(K)) \\
\text { subject to } & \left\|H_{z w}(\lambda)\right\|_{\infty}^{2}<\mu
\end{array}
$$

The mentioned problem is non-convex because of the noncovexity of the cardinality operator and the non-convex fixed-order $H_{\infty}$ dynamic output feedback controller synthesis problem. In the next subsections, a convex relaxation of the cardinality and an inner convex approximation of the $H_{\infty}$ constraints are presented.

\subsection{Convex Relaxation of Cardinality}

To reduce the amount of information exchange between subsystems and sub-controllers in an interconnected system, matrix $\mathcal{Z}(K)$ in $(12)$ should be sparse. The sparsity requirements are expressed in terms of the cardinality which is non-convex. It has been shown that the non-convex cardinality minimization can be relaxed by the convex one-norm $\left(\ell_{1}\right)$ minimization [33]. In fact, one-norm is the convex envelope of the cardinality [34]. 
To have a better approximation of the cardinality, the weighted $\ell_{1}$ norm is used [33]. Therefore, the objective function in (18) can be written as:

$$
J=\|W * \mathcal{Z}(K)\|_{1}
$$

where $W=\left[w_{i j}\right]$ is the matrix of weights. Matrix $J$ is defined as follows:

$$
J=\sum_{i=1}^{N} \sum_{j=1}^{N} w_{i j}\left\|k_{i j}\right\|_{1}
$$

where $k_{i j}=\left[\begin{array}{ll}A_{c_{i j}} & B_{c_{i j}} \\ C_{c_{i j}} & D_{c_{i j}}\end{array}\right]$ and $w_{i j} \geq 0$ is the $i j^{\text {th }}$ entry of $W$ which is chosen to be inversely proportional to $\left\|k_{i j}\right\|_{1}$, i.e.

$$
w_{i}= \begin{cases}1 /\left\|k_{i j}\right\|_{1}, & \text { if }\left\|k_{i j}\right\|_{1} \neq 0 \\ \infty, & \text { if }\left\|k_{i j}\right\|_{1}=0\end{cases}
$$

then the weighted $\ell_{1}$ norm and the cardinality operator coincide. However, since the weights depend on the unknown controller parameters, the above strategy cannot be implemented. An iterative algorithm for choosing the weighting matrix $W$ in which the weights are determined and updated through the solution of the weighted $\ell_{1}$ norm in the previous iteration has been developed in [33].

\subsection{Inner Convex Approximation of Fixed-order Stabilizing Controllers}

In this subsection, a convex set of fixed-order stabilizing controllers is given. The results are summarized in Theorem 1.

Theorem 1. Suppose that a slack matrix $M$ and a non-singular matrix $T$ are given. Then, the fixed-order controller of (9) stabilizes the polytopic system described by (4) if there exist Lyapunov matrices $P^{l}>0$ such that

$$
\left[\begin{array}{cc}
M^{T} P^{l}+P^{l} M & \star \\
P^{l}-M+T^{-1} A^{l} T & -2 I
\end{array}\right]<0
$$

for $l=1,2, \ldots, q$.

Proof. Convex combination of (22) for all $\lambda \in \Lambda$ leads to the following inequalities:

$$
\left[\begin{array}{cc}
M^{T} P(\lambda)+P(\lambda) M & \star \\
P(\lambda)-M+T^{-1} A(\lambda) T & -2 I
\end{array}\right]<0
$$


where $A(\lambda)=\sum_{l=1}^{q} \lambda_{l} A^{l}, P(\lambda)=\sum_{l=1}^{q} \lambda_{l} P^{l}$, and $\lambda \in \Lambda$.

Based on KYP Lemma, the inequality (23) indicates that the following transfer function matrix is strictly positive real (SPR) with Lyapunov matrix $P(\lambda)$ :

$$
H(s)=\left[\begin{array}{c|l}
M & I \\
\hline M-T^{-1} A(\lambda) T & I
\end{array}\right]
$$

It can be easily shown that the SPRness of $H(s)$ implies that $H^{-1}(s)$ with the following realization is also SPR with the same Lyapunov matrix $P(\lambda)$.

$$
H^{-1}(s)=\left[\begin{array}{c|c}
T^{-1} A(\lambda) T & I \\
\hline-M+T^{-1} A(\lambda) T & I
\end{array}\right]
$$

The SPRness of $H^{-1}$ with $P(\lambda)$ implies that matrix $T^{-1} A(\lambda) T$ is stable with Lyapunov matrix $P(\lambda)$. As a result, the closed-loop state matrix $A(\lambda)$ is stable with the linearly parameter-dependent Lyapunov matrix $T^{-T} P(\lambda) T^{-1}$.

Remark: One of the performance specifications in control theory is to optimize the spectral abscissa of the closed-loop system. This problem is reformulated as the following optimization problem:

$$
\begin{array}{ll}
\min _{K, P^{l}} & \beta \\
\text { s.t. } & {\left[\begin{array}{cc}
M^{T} P^{l}+P^{l} M-2 \beta P^{l} & \star \\
P^{l}-M+T^{-1} A^{l} T & -2 I
\end{array}\right]<0 ; \quad \beta<0} \\
& P^{l}>0
\end{array}
$$

for $l=1, \ldots, q$. The minimum value of $\beta$ can be obtained by a bisection algorithm.

\subsection{Inner Convex Approximation of Fixed-order $H_{\infty}$ Controllers}

In this subsection, an LMI representation of fixed-order $H_{\infty}$ controller synthesis of continuous-time LTI systems with polytopic uncertainty is provided.

Theorem 2. Suppose that two slack matrices $M$ and $T$ are given. Then, the fixed-order controller of (9) guarantees the robust stability and the robust performance $\left\|H_{z w}(\lambda)\right\|_{\infty}^{2}<\mu$ of the closed-loop system given in (14)-(17) if there exist Laypunov matrices $P^{l}>0$ such that

$$
\left[\begin{array}{cccc}
M^{T} P^{l}+P^{l} M & \star & \star & \star \\
P^{l}-M+T^{-1} A^{l} T & -2 I & \star & \star \\
0 & \left(T^{-1} B^{l}\right)^{T} & -I & \star \\
C^{l} T & 0 & D^{l} & -\mu I
\end{array}\right]<0
$$


for $l=1, \ldots, q$.

Proof. See Appendix A.

The inequalities given in (27) are LMIs in terms of output feedback controller parameters $\left(A_{c}, B_{c}, C_{c}, D_{c}\right), \mu$, and matrices $P^{l} ; l=1, \ldots, q$.

Lemma 1. The following set of inequalities is equivalent to (27):

$$
\left[\begin{array}{cccc}
A^{l^{T}} P_{T}^{l}+P_{T}^{l} A^{l} & \star & \star & \star \\
P_{T}^{l}+M_{T}-X A^{l} & -2 X & \star & \star \\
-B^{l^{T}} M_{T}+B^{l^{T}} X A^{l} & B^{l^{T}} X & -I & \star \\
C^{l} & 0 & D^{l} & -\mu I
\end{array}\right]<0
$$

where $M_{T}, P_{T}^{l}$, and $X$ are defined as follows:

$$
\begin{aligned}
M_{T} & =T^{-T} M T^{-1} \\
P_{T}^{l} & =T^{-T} P^{l} T^{-1} \\
X & =T^{-T} T^{-1}
\end{aligned}
$$

for $l=1, \ldots, q$.

Proof. See Appendix B.

The non-convexity of the fixed-order controller design problem can be overcome by means of the slack matrices $M$ and $T$. However, one of the main sources of conservatism in the proposed approach is the choice of these matrices. In the following, a heuristic approach for choosing the slack matrices is given.

Generally, one approach to the choice of the slack matrices $M$ and $T$ is to use a set of initial fixed-order stabilizing controllers designed for each vertex of the polytopic system using the existing methods in the literature, e.g. $[35,36,37,38,39,40]$.

Suppose that $\left(\bar{A}^{l}, \bar{B}^{l}, \bar{C}^{l}, \bar{D}^{l}\right)$ is the closed-loop state space realization of the $l^{\text {th }}$ vertex with its corresponding controller $K^{l}$. Then, matrices $M$ and $T$ can be obtained as follows:

$$
\begin{aligned}
T & =(\operatorname{chol}(X))^{-1} \\
M & =T^{T} M_{T} T
\end{aligned}
$$


where chol denotes Cholesky factorization and $\left(M_{T}, X\right)$ is a feasible solution of the following convex optimization problem:

$$
\begin{aligned}
& \min _{M_{T}, X, P_{T}^{l}, \mu} \mu \\
& \text { s.t. } \quad\left[\begin{array}{cccc}
\bar{A}^{l} P_{T}^{l}+P_{T}^{l} \bar{A}^{l} & \star & \star & \star \\
P_{T}^{l}+M_{T}-X \bar{A}^{l} & -2 X & \star & \star \\
-\bar{B}^{l^{T}} M_{T}+\bar{B}^{l^{T}} X \bar{A}^{l} & \bar{B}^{l^{T}} X & -I & \star \\
\bar{C}^{l} & 0 & \bar{D}^{l} & -\mu I
\end{array}\right]<0 \\
& P_{T}^{l}>0 \\
& l=1, \ldots, q
\end{aligned}
$$

\subsection{Convex Set of Fixed-order Decentralized/Distributed $H_{\infty}$ Controllers}

The problem of fixed-order decentralized/distributed $H_{\infty}$ control of LTI interconnected systems subject to polytopic uncertainty can be solved by the following convex optimization problem:

$$
\begin{aligned}
& \min _{K, P^{l}, \mu} \mu+\alpha\|W * \mathcal{Z}(K)\|_{1} \\
& {\left[\begin{array}{cccc}
M^{T} P^{l}+P^{l} M & \star & \star & \star \\
P^{l}-M+T^{-1} A^{l} T & -2 I & \star & \star \\
0 & \left(T^{-1} B^{l}\right)^{T} & -I & \star \\
C^{l} T & 0 & D^{l} & -\mu I
\end{array}\right]<0} \\
& \quad P^{l}>0 \\
& \quad l=1, \ldots, q
\end{aligned}
$$

where $\alpha$ determines a trade-off between the number of communication links in the distributed control and the $H_{\infty}$ performance criterion. In following, a systematic algorithm for the problem of fixed-order decentralized/distributed $H_{\infty}$ controller design of LTI polytopic systems is given.

\subsection{Fixed-order Decentralized/Distributed $H_{\infty}$ Controller Design Algorithm}

In this subsection, an iterative LMI-based algorithm for the problem of fixed-order sparse $H_{\infty}$ controller design is presented. The iterative procedure can be summarized by the following steps:

Step 1 (Initialization): Design some initial controllers for each vertex of the polytope $\left(K^{l[0]}\right)$. Put the iteration number $h=1$, a small tolerance 
for $\epsilon>0$, and $w_{i j}^{[1]}=1, i, j=1, \ldots, N$. Determine $\alpha$ based on the desired $H_{\infty}$ performance and the sparsity of the controller.

Step 2: Solve the convex optimization problem given in (31) and determine $M_{T}^{[h]}$ and $X^{[h]}$.

Step 3: Find the slack matrices $M^{[h]}$ and $T^{[h]}$ from (29).

Step 4: Solve the convex optimization problem given in (32) to obtain a sparse fixed-order $H_{\infty}$ controller $K^{[h]}$. $K^{[h]}$.

Step 5: Find $\left\|k_{i j}\right\|_{1}^{[h]}, i, j=1, \ldots, N$ based on the current controller

Step 6: Update the $i j^{\text {th }}$ elements of the weighting matrix $W^{[h+1]}$ :

$$
w_{i j}^{[h+1]}=\left\{\begin{array}{cl}
\frac{1}{\left\|k_{i j}\right\|_{1}^{[h]}+\epsilon}, & i \neq j \\
0, & i=j
\end{array}\right.
$$

for $i, j=1, \ldots, N$.

Step 7: Terminate on convergence or when maximum number of iterations $h_{\max }$ reaches. Otherwise, use the obtained controller in Step 4 as an initial controller $\left(K^{l[h]} \leftarrow K^{[h]} ; l=1, \ldots, q\right)$ and go to Step 2 with $h \leftarrow h+1$.

Step 8 (Polishing): Design a fixed-structure $H_{\infty}$ controller by solving the optimization problem in (32), where $\alpha=0$ is considered.

Remark: It can be easily shown that the fixed-order $H_{\infty}$ controller design algorithm $(\alpha=0)$ leads to monotonic convergence of the upper bound of the $H_{\infty}$ norm. The proof stems from the fact that (27) and (28) are equivalent. Therefore, for $h>1, K^{[h-1]}$ and $\mu^{[h-1]}$ are always feasible solutions to the optimization problem in Step 4. On the other hand, $M_{T}^{[h]}, X^{[h]}, \mu^{[h]}$ are always solutions to the optimization problem in Step 2 at iteration $h+1$. Thus, $\mu^{[h+1]} \leq \mu^{[h]}$ which confirms that the upper bound of the $H_{\infty}$ norm is not increasing. Weighted $\ell_{1}$ norm also converges to a local minimum [33]. Nevertheless, the convergence of the proposed algorithm cannot be guaranteed.

In the next section, the proposed results are applied to the problem of voltage control of islanded inverter-interfaced microgrids.

\section{INVERTER-INTERFACED MICROGRIDS}

\subsection{Microgrid System Description}

Consider an islanded inverter-interfaced microgrid consisting of $N \mathrm{DG}$ units. Each DG unit is modeled by a DC voltage source, a voltage-source 


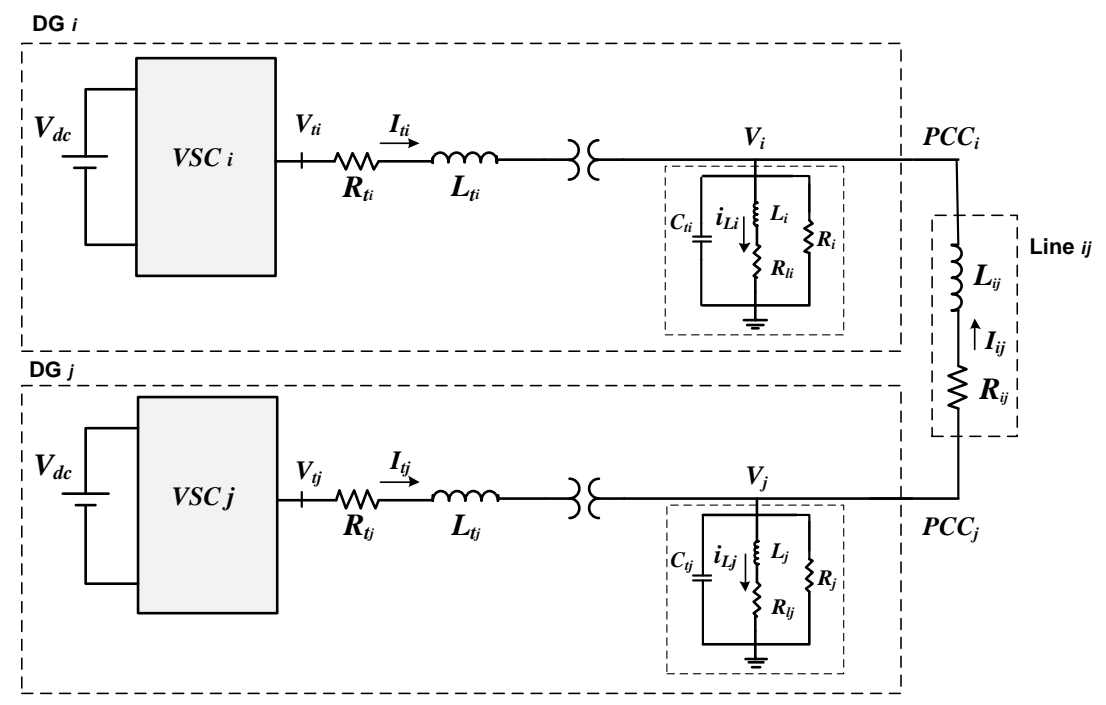

Figure 1: Configuration of two DG units connected via line $i j$

converter (VSC), a series RL filter, a step-up transformer with transformation ratio $k$, and a local load modeled by a three-phase parallel RLC network. For the sake of simplicity, first we consider the configuration of a microgrid system with two DG units as shown in Fig. 1. However, the proposed voltage control method in this paper is general and it can be applied to the microgrids composed of $N$ DGs. The system is described by the following dynamical equations in $d q$-frame:

$$
\begin{gathered}
\text { DG } i\left\{\begin{array}{l}
\frac{d V_{i, d q}}{d t}+j \omega_{0} V_{i, d q}=-\frac{1}{R_{i} C_{t i}} V_{i, d q}+\frac{k}{C_{t i}} I_{t i, d q}-\frac{1}{C_{t i}} i_{L_{i}, d q}+\frac{1}{C_{t i}} I_{i j, d q} \\
\frac{d I_{t i}, d q}{d t}+j \omega_{0} I_{t i, d q}=-\frac{k}{L_{t i}} V_{i, d q}-\frac{R_{t i}}{L_{t i}} I_{t i, d q}+\frac{1}{L_{t i}} V_{t i, d q} \\
\frac{d L_{i, d q}}{d t}+j \omega_{0} i_{L_{i}, d q}=\frac{1}{L_{i}} V_{i, d q}-\frac{R_{L_{i}}}{L_{i}} i_{L_{i}, d q}
\end{array}\right. \\
\text { DG } j\left\{\begin{array}{l}
\frac{d V_{j, d q}}{d t}+j \omega_{0} V_{j, d q}=-\frac{1}{R_{j} C_{t j}} V_{j, d q}+\frac{k}{C_{t j}} I_{t j, d q}-\frac{1}{C_{t j}} i_{L_{j}, d q}-\frac{1}{C_{t j}} I_{i j, d q} \\
\frac{d t_{j, d q}}{d t}+j \omega_{0} I_{t j, d q}=-\frac{k}{L_{t j}} V_{j, d q}-\frac{R_{t j}}{L_{t j}} I_{t j, d q}+\frac{1}{L_{t j}} V_{t j, d q} \\
\frac{d i_{j}, d q}{d t}+j \omega_{0} i_{L_{j}, d q}=\frac{1}{L_{j}} V_{j, d q}-\frac{R_{l j}}{L_{j}} i_{L_{j}, d q}
\end{array}\right.
\end{gathered}
$$




$$
\text { Line } i j: \quad \frac{d I_{i j, d q}}{d t}+j \omega_{0} I_{i j, d q}=-\frac{R_{i j}}{L_{i j}} I_{i j, d q}+\frac{1}{L_{i j}} V_{j, d q}-\frac{1}{L_{i j}} V_{i, d q}
$$

where $\left(V_{i, d q}, V_{j, d q}\right),\left(I_{t i, d q}, I_{t j, d q}\right),\left(i_{L_{i}, d q}, i_{L_{j}, d q}\right),\left(V_{t i, d q}, V_{t j, d q}\right)$, and $I_{i j, d q}$ respectively are the $d q$ components of the load voltage at PCCs, the current filters, the load inductance currents, the VSC terminal voltages, and the transmission line current.

To obtain the above dynamical equations, the system is mathematically described in the $a b c$-frame. Then, the mathematical model is transformed to $\alpha \beta$-frame using $x_{\alpha \beta}=x_{a}+x_{b} e^{-j(2 \pi / 3)}+x_{c} e^{j(2 \pi / 3)}$. Finally, they are transformed to the $d q$-frame using $x_{d q}=\sqrt{2 / 3} x_{\alpha \beta} e^{j \theta}$, where $\theta$ is the transformation angle. It should be noted that the dc-side of VSC is modeled by an ideal voltage source.

The microgrid system in Fig. 1 can be presented as a linear time-invariant system by the following state space equations:

$$
\begin{aligned}
& {\left[\begin{array}{c}
\dot{x}_{i}(t) \\
\dot{x}_{l_{i j}}(t) \\
\dot{x}_{j}(t)
\end{array}\right]=\left[\begin{array}{c:c:c}
A_{i} & A_{l i j} & 0 \\
\hdashline-A_{l j, i j} & \bar{A}_{l, i j} & A_{l j, i j} \\
\hdashline 0 & -A_{l j i} & A_{j}
\end{array}\right]\left[\begin{array}{c}
x_{i}(t) \\
x_{l_{i j}}(t) \\
x_{j}(t)
\end{array}\right]+\left[\begin{array}{c:c}
B_{i} & 0 \\
\hdashline 0 & 0 \\
\hdashline 0 & B_{j}
\end{array}\right]\left[\begin{array}{c}
u_{i}(t) \\
u_{j}(t)
\end{array}\right]} \\
& {\left[\begin{array}{l}
y_{i}(t) \\
y_{j}(t)
\end{array}\right]=\left[\begin{array}{c:c:c}
C_{i} & 0 & 0 \\
\hdashline 0 & 0 & C_{j}
\end{array}\right]\left[\begin{array}{c}
x_{i}(t) \\
x_{l_{i j}}(t) \\
x_{j}(t)
\end{array}\right]}
\end{aligned}
$$

where $x_{i}=\left[\begin{array}{llllll}V_{i, d} & V_{i, q} & I_{t i, d} & I_{t i, q} & i_{L_{i, d}} & i_{L_{i, q}}\end{array}\right]^{T}, x_{l_{i j}}=\left[\begin{array}{lll}I_{i j, q} & I_{i j, q}\end{array}\right]^{T}, x_{j}=$ $\left[\begin{array}{llllll}V_{j, d} & V_{j, q} & I_{t j, d} & I_{t j, q} & i_{L_{j}, d} & i_{L_{j}, q}\end{array}\right]^{T}, u_{i}=\left[\begin{array}{ll}V_{t i, d} & V_{t i, q}\end{array}\right]^{T}, u_{j}=\left[\begin{array}{ll}V_{t j, d} & V_{t j, q}\end{array}\right]^{T}$, $y_{i}=\left[\begin{array}{ll}V_{i, d} & V_{i, q}\end{array}\right]^{T}, y_{j}=\left[\begin{array}{ll}V_{j, d} & V_{j, q}\end{array}\right]^{T}$, and

$$
A_{i}=\left[\begin{array}{cccccc}
-\frac{1}{R_{i} C_{t i}} & \omega_{0} & \frac{k}{C_{t i}} & 0 & -\frac{1}{C_{t i}} & 0 \\
-\omega_{0} & -\frac{1}{R_{i} C_{t i}} & 0 & \frac{k}{C_{t i}} & 0 & -\frac{1}{C_{t i}} \\
-\frac{k}{L_{t i}} & 0 & -\frac{R_{t i}}{L_{t i}} & \omega_{0} & 0 & 0 \\
0 & -\frac{k}{L_{t i}} & -\omega_{0} & -\frac{R_{t i}}{L_{t i}} & 0 & 0 \\
\frac{1}{L_{i}} & 0 & 0 & 0 & -\frac{R_{l i}}{L_{i}} & \omega_{0} \\
0 & \frac{1}{L_{i}} & 0 & 0 & -\omega_{0} & -\frac{R_{l i}}{L_{i}}
\end{array}\right], \quad A_{l i j}=\left[\begin{array}{cc}
\frac{1}{C_{t i}} & 0 \\
0 & \frac{1}{C_{t i}} \\
0 & 0 \\
0 & 0 \\
0 & 0 \\
0 & 0
\end{array}\right]
$$




$$
\begin{aligned}
& A_{j}=\left[\begin{array}{cccccc}
-\frac{1}{R_{j} C_{t j}} & \omega_{0} & \frac{k}{C_{t j}} & 0 & -\frac{1}{C_{t j}} & 0 \\
-\omega_{0} & -\frac{1}{R_{j} C_{t j}} & 0 & \frac{k}{C_{t j}} & 0 & -\frac{1}{C_{t j}} \\
-\frac{k}{L_{t j}} & 0 & -\frac{R_{t j}}{L_{t j}} & \omega_{0} & 0 & 0 \\
0 & -\frac{k}{L_{t j}} & -\omega_{0} & -\frac{R_{t j}}{L_{t j}} & 0 & 0 \\
\frac{1}{L_{j}} & 0 & 0 & 0 & -\frac{R_{l j}}{L_{j}} & \omega_{0} \\
0 & \frac{1}{L_{j}} & 0 & 0 & -\omega_{0} & -\frac{R_{l j}}{L_{j}}
\end{array}\right], \quad A_{l j i}=\left[\begin{array}{cc}
\frac{1}{C_{t j}} & 0 \\
0 & \frac{1}{C_{t j}} \\
0 & 0 \\
0 & 0 \\
0 & 0 \\
0 & 0
\end{array}\right] \\
& A_{l, i j}=\left[\begin{array}{cc}
-\frac{R_{i j}}{L_{i j}} & \omega_{0} \\
-\omega_{0} & -\frac{R_{i j}}{L_{i j}}
\end{array}\right], \quad A_{l j, i j}=\left[\begin{array}{cccccc}
\frac{1}{L_{i j}} & 0 & 0 & 0 & 0 & 0 \\
0 & \frac{1}{L_{i j}} & 0 & 0 & 0 & 0
\end{array}\right] \\
& B_{i}=\left[\begin{array}{cc}
0 & 0 \\
0 & 0 \\
\frac{1}{L_{t i}} & 0 \\
0 & \frac{1}{L_{t i}} \\
0 & 0 \\
0 & 0
\end{array}\right], \quad B_{j}=\left[\begin{array}{cc}
0 & 0 \\
0 & 0 \\
\frac{1}{L_{t j}} & 0 \\
0 & \frac{1}{L_{t j}} \\
0 & 0 \\
0 & 0
\end{array}\right] \\
& C_{i}=C_{j}=\left[\begin{array}{cccccc}
1 & 0 & 0 & 0 & 0 & 0 \\
0 & 1 & 0 & 0 & 0 & 0
\end{array}\right]
\end{aligned}
$$

\subsection{Islanded Microgrids with $N$ DG Units}

The state space model in (37) can be extended to the islanded microgrids composed of $N$ DG units with a parallel topology. The overall microgrid system $G(s)$ is described as follows:

$$
\begin{aligned}
\dot{x}_{g}(t) & =A_{g} x_{g}(t)+B_{g} u(t) \\
y(t) & =C_{g} x_{g}(t)
\end{aligned}
$$

where

$$
\begin{aligned}
x_{g} & =\left[\begin{array}{llllll}
x_{1}^{T} & x_{l_{12}}^{T} & x_{2}^{T} & x_{l_{23}}^{T} & \ldots & x_{N}^{T}
\end{array}\right]^{T} \\
u & =\left[\begin{array}{lll}
u_{1}^{T} & \ldots & u_{N}^{T}
\end{array}\right]^{T} \\
y & =\left[\begin{array}{lll}
y_{1}^{T} & \ldots & y_{N}^{T}
\end{array}\right]^{T}
\end{aligned}
$$

and the state space matrices are given: 


$$
\begin{aligned}
A_{g} & =\left[\begin{array}{ccccccc}
A_{1} & A_{l 12} & 0 & 0 & 0 & \ldots & 0 \\
-A_{l 2,12} & A_{l, 12} & A_{l 2,12} & 0 & 0 & \ldots & 0 \\
0 & -A_{l 21} & A_{2} & A_{l 23} & 0 & \ldots & 0 \\
0 & 0 & -A_{l 3,23} & A_{l, 23} & A_{l 3,23} & \ldots & 0 \\
\vdots & \vdots & \vdots & \vdots & \vdots & \ddots & \vdots \\
0 & 0 & 0 & 0 & 0 & \ldots & A_{N}
\end{array}\right] \\
B_{g} & =\left[\begin{array}{ccccc}
B_{1} & 0 & 0 & \ldots & 0 \\
0 & 0 & 0 & \ldots & 0 \\
0 & B_{2} & 0 & \ldots & 0 \\
0 & 0 & 0 & \ldots & 0 \\
0 & 0 & B_{3} & \ldots & 0 \\
\vdots & \vdots & \vdots & \ddots & \vdots \\
0 & 0 & 0 & \ldots & B_{N}
\end{array}\right], C_{g}=\left[\begin{array}{ccccccc}
C_{1} & 0 & 0 & 0 & 0 & \ldots & 0 \\
0 & 0 & C_{2} & 0 & 0 & \ldots & 0 \\
0 & 0 & 0 & 0 & C_{3} & \ldots & 0 \\
\vdots & \vdots & \vdots & \vdots & \vdots & \ddots & \vdots \\
0 & 0 & 0 & 0 & 0 & \ldots & C_{N}
\end{array}\right]
\end{aligned}
$$

where $A_{i}, A_{l i j}, A_{l j, i j}, A_{l, i j}, B_{i}$, and $C_{i}$ are define in (38)-(42) for $i, j=$ $1, \ldots, N$.

\subsection{Islanded Microgrids with Polytopic-type Uncertainty}

The parametric uncertainty of the microgrid system in Fig. 1 arises from the fact that the RLC load parameters of DG unit $i$ can vary based on consumers demands. For the sake of simplicity, the following definitions are used.

$$
\theta_{i}^{1}=\frac{1}{R_{i}}, \quad \theta_{i}^{2}=\frac{1}{L_{i}}, \quad \theta_{i}^{3}=\frac{1}{C_{t i}}
$$

It is also assumed that the RLC load parameters are bounded within the maximum and minimum values as $R_{i_{\min }} \leq R_{i} \leq R_{i_{\max }}, L_{i_{\min }} \leq L_{i} \leq L_{i_{\max }}$, and $C_{i_{\text {min }}} \leq C_{t i} \leq C_{i_{\max }}$ which represents a cube in which the load parameters are allowed to change. In the general case of $N$ DG units, it is a hyper-cube with $q=2^{n_{\theta}}$ vertices, where $n_{\theta}$ is the number of uncertain load parameters. It can be shown that the image of this hyper-cube in the space of the elements of the matrix $A_{g}$ is inside a polytope of $q=2^{n_{\theta}}$ vertices. This polytope covers the whole uncertainty in the RLC load parameters and is defined as the convex combination of the vertices $A_{g}^{l}, l=1, \ldots, q[41]$ :

$$
A_{g}(\lambda)=\sum_{l=1}^{q} \lambda_{l} A_{g}^{l}, \quad \lambda_{l} \in \Lambda
$$




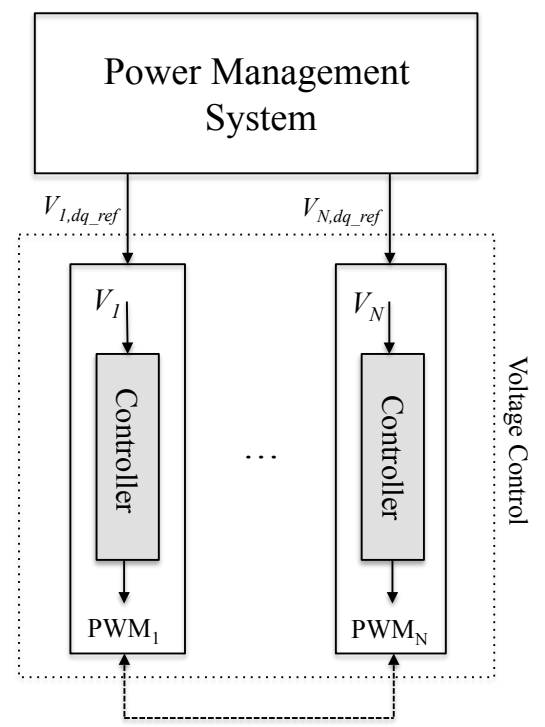

GPS Time-reference Signal

Figure 2: Block diagram of the islanded microgrid control strategy

where vertices $A_{g}^{l}$ are obtained based on the maximum and minimum values of the RLC load parameters.

\subsection{Islanded Microgrid Control System}

Consider a schematic diagram of the microgrid control strategy composed of a power management system (PMS), local voltage controllers of DG units, and a frequency control scheme in Fig. 2.

A power management strategy is required for reliable and efficient operation of a microgrid system with multiple DG units, particularly in the islanded mode of operation [42]. The main function of the power management system (PMS) is to maintain an optimal operating point for the microgrid. PMS assigns the active and reactive power set points for the DG units to (i) properly share the real and reactive power among the DG units based on either a cost function associated with each DG unit or a market signal [23], (ii) appropriately respond to the microgrid disturbances and major changes [43], (iii) balance the microgrid power, and (iv) provide the resynchronization of the microgrid system with the main grid, if required [43]. The set points are then transmitted to the local voltage controllers of the DG units. The local controllers measure the voltage at their corresponding PCC or the ac- 
tive/reactive output power of their own DG unit and then enable the voltage tracking according to the received reference set points [26].

The frequency of the islanded microgrid system is controlled in the openloop. To this end, each DG unit includes an oscillator which generates $\theta(t)=\int_{0}^{t} \omega_{0} d \tau$, where $\omega_{0}=2 \pi f_{0}$ and $f_{0}$ is the nominal frequency of the microgrid. The phase-angle waveform $\theta(t)$ is employed for $d q / a b c(a b c / d q)$ transformations. The DG units are then synchronized by a global synchronization signal that is communicated to the oscillators of DG units through the global positioning system (GPS) [23].

The voltage set points are communicated from PMS to local controllers of the DG units and transformed to the $d q$-frame based on the phase-angle signal $\theta(t)$ generated by their internal oscillator. The main objective is to develop a robust voltage controller for the islanded operation of the inverterinterfaced microgrids with load parameter uncertainties given in (46).

\subsection{Robust Fixed-order Decentralized Voltage Control}

A fixed-order voltage controller for the islanded microgrid system whose dynamical equations are given in (43)-(45) with polytopic-type uncertainty given in (46) is sought to satisfy the following performance criteria:

- The closed-loop system must be asymptotically stable for the whole polytope.

- The closed-loop polytopic system should be able to asymptotically track all step reference voltage signals $\left(y_{\text {ref }}(t)\right)$.

- The closed-loop response to step reference voltage signals should be fast with small overshoot for all values of the load parameters within the prespecified uncertainty ranges.

- Each local controller uses the minimum information exchange and communication among DG units and their local controllers.

- The local controllers are structurally simple (low-order control design).

- The coupling among the output channels should be small.

To achieve all above mentioned conditions, in following, a fixed-order 2DOF sparse controller with integral action is designed. The dynamics of 
the controller $K(s)$ are given by:

$$
\begin{array}{r}
\dot{x}_{c}(t)=A_{c} x_{c}(t)+\left[\begin{array}{ll}
B_{c_{1}} & B_{c_{2}}
\end{array}\right]\left[\begin{array}{c}
y(t) \\
y_{\text {ref }}(t)
\end{array}\right] \\
u(t)=C_{c} x_{c}(t)+\left[\begin{array}{ll}
D_{c_{1}} & D_{c_{2}}
\end{array}\right]\left[\begin{array}{c}
y(t) \\
y_{\text {ref }}(t)
\end{array}\right]
\end{array}
$$

where $x_{c}(t)$ is the states of the controller and matrices $A_{c}, B_{c_{1}}, B_{c_{2}}, C_{c}, D_{c_{1}}$, and $D_{c_{2}}$ are of appropriate dimensions. The controller is a solution of the following optimization problem:

$$
\begin{array}{ll}
\min _{A_{c}, B_{c_{1}}, B_{c_{2}}, C_{c}, D_{c_{1}}, D_{c_{2}}} & \mu+\alpha\|W * \mathcal{Z}(K)\|_{1} \\
\text { subject to } & \left\|W_{s} S(\lambda)\right\|_{\infty}^{2}<\mu
\end{array}
$$

where $\mathcal{Z}(K)$ is defined in (12). Transfer functions $S(\lambda)=(I+G(\lambda) K)^{-1}$ and $W_{s}$ are sensitivity function and a weighting filter designed based on the desired time-domain performance [44]. The positive scalar $\alpha$ characterizes the emphasis on the tracking dynamics and the sparsity of the controller architecture.

The weighting filter $W_{s}$ is responsible to shape the sensitivity function $S$ and provides the desirable performance characteristics of the closed-loop system. A common choice of $W_{s}$ is given as follows [27]:

$$
W_{s}(s)=\operatorname{diag}\left(\left[\begin{array}{cc}
\frac{s}{M_{s_{i}}}+\omega_{B_{i}}^{*} & 0 \\
s+\omega_{B_{i}}^{*} \epsilon_{i} & \frac{s}{M_{s_{i}}}+\omega_{B_{i}}^{*} \\
0 & \frac{s+\omega_{B_{i}}^{*} \epsilon_{i}}{0}
\end{array}\right]\right)
$$

where $\omega_{B_{i}}^{*}$ is approximately the desired closed-loop bandwidth, $\epsilon_{i}$ is the maximum tracking steady state error, and $M_{s_{i}} \geq 1$ is the maximum peak value of $S$. The choice of $\epsilon_{i}<<1$ ensures approximate integral action $S(0) \approx 0$ [27]. A large value of $\omega_{B_{i}}^{*}$ leads to a faster response for output $i$. However, there always exists a trade-off between the speed of the closed-loop system response and the sensitivity of the closed-loop system with respect to the measurement noise. Therefore, to have an acceptable dynamic response of the microgrid system in terms of step signal tracking and robustness to the measurement noise, the parameters of the weighting filter $W_{s}(s)$ are selected as follows:

$$
\omega_{B_{i}}^{*}=30, \quad M_{s_{i}}=1.5, \quad \epsilon_{i}=3.33 e-4
$$


Table 1: Parameters of islanded single-DG microgrid

\begin{tabular}{|c|c|}
\hline Filter parameters & $R_{t}=37.7 \mathrm{~m} \Omega, \quad L_{t}=5 \mathrm{mH}$ \\
DC bus voltage & $V_{d c}=340 \mathrm{~V}$ \\
VSC rated power & $S_{V S C}=10 \mathrm{KVA}$ \\
PWM carrier frequency & $f_{s w}=10 \mathrm{KHz}$ \\
\hline Load nominal resistance & $R_{n o m}=23 \Omega$ \\
Load nominal inductance & $L_{n o m}=5 \mathrm{mH}$ \\
Load nominal capacitance & $C_{n o m}=850 \mu \mathrm{F}$ \\
Inductor quality factor & $q_{l}=120$ \\
\hline System nominal frequency & $f_{0}=60 \mathrm{~Hz}\left(\omega_{0}=2 \pi f_{0}\right)$ \\
\hline
\end{tabular}

$\omega_{B_{i}}^{*}=30$ is chosen to have a rise-time of about $50 \mathrm{~ms}, M_{s_{i}}=1.5$ is chosen to have a peak value of $\left\|S_{i}\right\|_{\infty} \leq M_{s_{i}}$ and $\epsilon_{i}=3.33 e-4$ is chosen to have very small steady state error with respect to a step input, i.e. less than $3.33 e-4$.

\subsection{Simulation and Experimental Results}

In this subsection, the performance of the proposed voltage control strategy is verified by a set of comprehensive simulation studies and is validated by means of experiments.

Scenario 1: To show different performance aspects of the proposed voltage control design technique and for the sake of simplicity, we consider a single-DG microgrid system which supplies a three-phase parallel RLC network whose parameters are given in Table 1 . It is assumed that the load resistance $R_{1}$ can vary within $\pm 80 \%$ of its nominal value $\left(R_{\text {nom }}\right)$. Moreover, the load parameters $L_{1}$ and $C_{t 1}$ are assumed to be bounded in the intervals $[0.5,1.5] \times L_{n o m}$ and $[0.5,1.5] \times C_{n o m}$, respectively, where $L_{n o m}$ and $C_{\text {nom }}$ are their nominal values.

The frequency of the islanded microgrid is controlled through an internal oscillator in the open-loop manner with $\omega_{0}=2 \pi f_{0}$. According to the fixedorder $H_{\infty}$ controller design procedure discussed in Subsection 3.5 in the case of $\alpha=0$, a robust sixth-order 2DOF voltage controller with integral action is designed: 


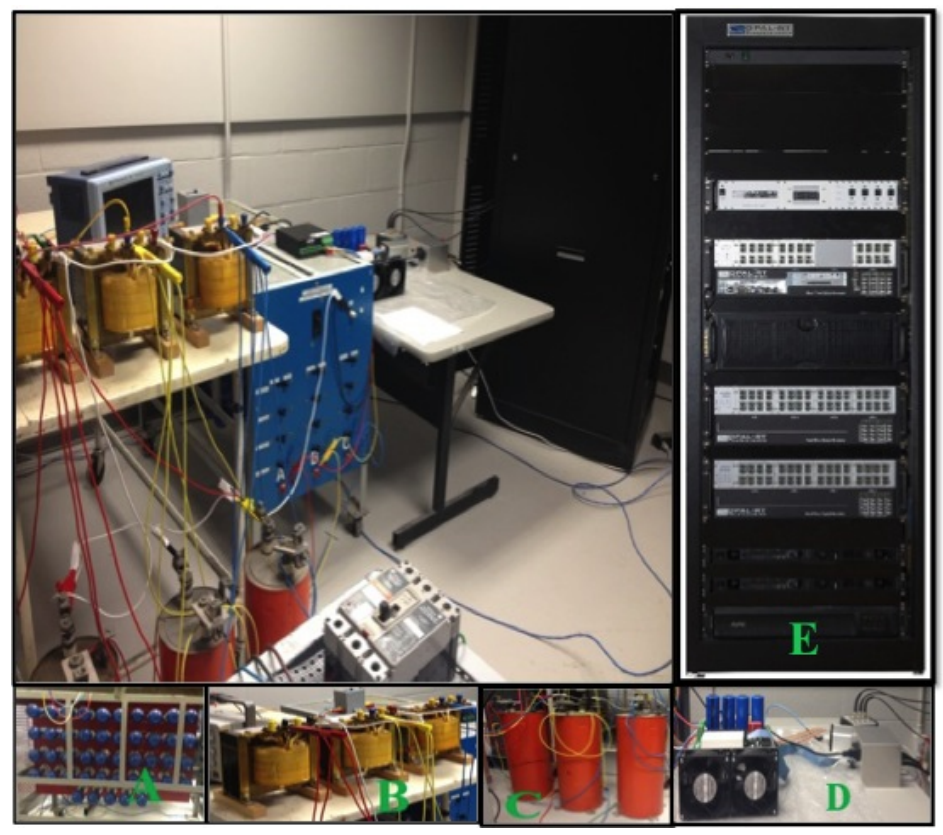

Figure 3: Experimental setup: (A) load resistance, (B) load inductance, (C) load capacitance, (D) three-phase converter and gating signal generator, and (E) OPAL-RT.

$$
\begin{aligned}
& A_{c}=\left[\begin{array}{cccccc}
0 & 114.187 & 200.576 & 0 & -3.384 e 3 & 2.687 e 3 \\
0 & -1.281 e 4 & 8.110 e 3 & 0 & -7.561 e 3 & 5.674 e 3 \\
0 & 964.293 & -1.227 e 4 & 0 & -2.328 e 3 & 1.779 e 3 \\
0 & 1.495 e 3 & -1.003 e 3 & 0 & 560.633 & -321.734 \\
0 & -1.581 e 3 & 2.471 e 3 & 0 & -3.086 e 4 & 2.430 e 4 \\
0 & -6.624 e 3 & 4.181 e 3 & 0 & -4.259 e 3 & -7.559 e 3
\end{array}\right], \quad C_{c}=\left[\begin{array}{cc}
14.552 & 25.395 \\
-1.387 e 3 & 53.608 \\
957.818 & 154.008 \\
-22.352 & 17.363 \\
-1.119 e 3 & -2.948 e 3 \\
793.861 & 2.359 e 3
\end{array}\right]^{T} \\
& B_{c_{1}}=\left[\begin{array}{cc}
-3.96 & -10.813 \\
-190.697 & -7.335 \\
-1.278 e 3 & -23.570 \\
20.591 & -15.528 \\
46.662 & -92.329 \\
-55.562 & -1.354 e 3
\end{array}\right], \quad B_{c_{2}}=\left[\begin{array}{cc}
20.817 & 3.304 \\
48.723 & -34.250 \\
28.047 & 2.704 \\
-11.663 & 29.727 \\
-24.629 & 1.592 \\
13.016 & 51.422
\end{array}\right] \\
& D_{c_{1}}=\left[\begin{array}{cc}
-20.298 & -4.549 \\
7.979 & -11.005
\end{array}\right], \quad D_{c_{2}}=\left[\begin{array}{cc}
6.238 & -5.233 \\
-1.149 & 1.927
\end{array}\right]
\end{aligned}
$$

The designed controller guarantees the robust stability as well as the robust performance criterion $\left\|W_{s} S(\lambda)\right\|_{\infty}<1.087$ in spite of the prespecified 
(a)

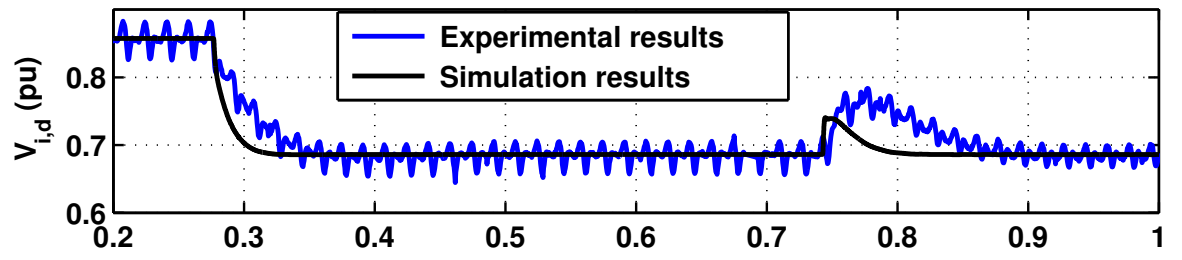

(b)

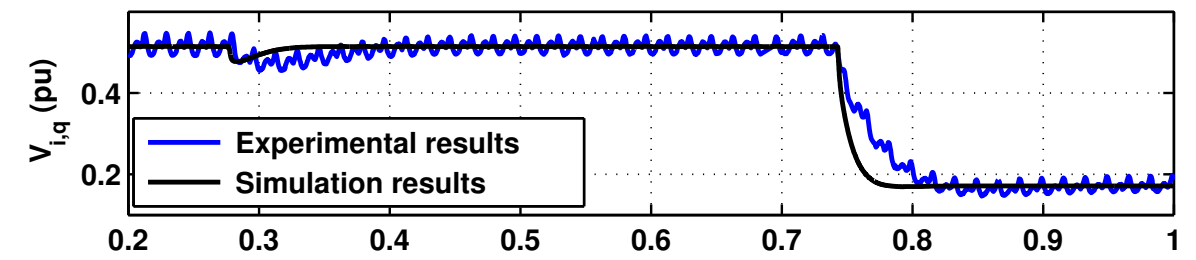

(c)

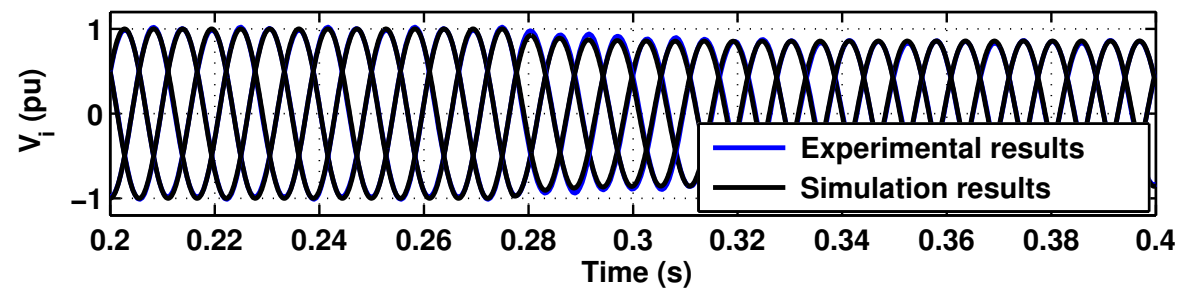

Figure 4: Experimental and simulation results of the islanded microgrid in voltage tracking (a) $d$-component of the load voltage, (b) $q$-component of the load voltage, and (c) instantaneous load voltages

load parameter uncertainties. Moreover, the controller provides the asymptotic tracking of all step reference inputs.

\section{Experimental Results}

In this part, the performance of the designed robust $H_{\infty}$ voltage controller is validated by means of an experimental test system with the parameters given in Table 1. The voltage controller given in (51) is implemented in the RT-LAB real-time platform of OPAL-RT Technologies ${ }^{1}$ with the sampling time of $9 \mu s$.

A photo of the laboratory experimental setup is shown in Fig. 3 which includes OPAL-RT, three-phase two-level converter, and three-phase $R L C$

\footnotetext{
${ }^{1}$ www.opal-rt.com
} 
(a)

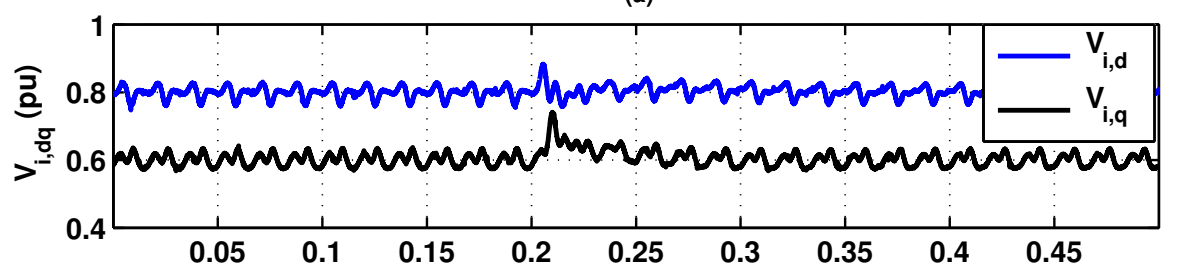

(b)

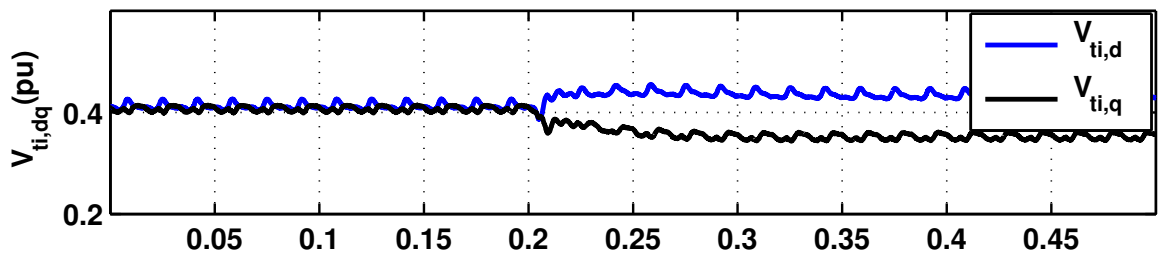

(c)

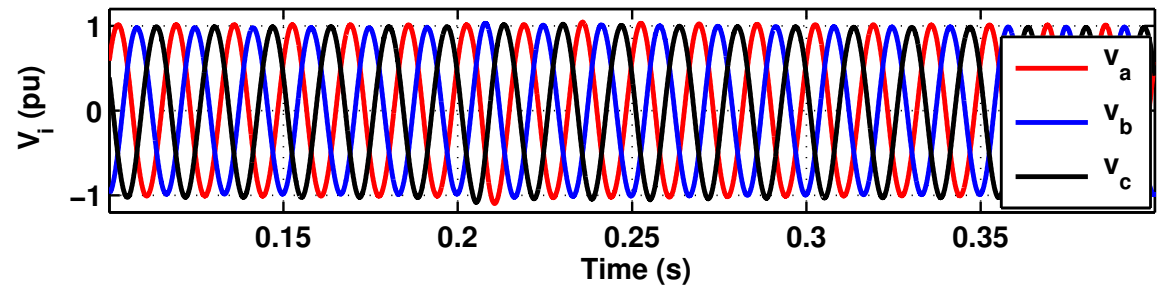

Figure 5: Dynamic responses of the experimental test system due to a resistive load change (a) $d q$-components of the load voltage, (b) control inputs, and (c) instantaneous load voltages

load. The performance of the control system is validated using several tests including voltage tracking and sudden changes in the load parameters. In all case studies, the system is assumed balanced and operates in the islanded mode.

The first test demonstrates the capability of the designed controller in reference signal tracking. The $d$-component of the reference voltage steps down to $0.686 \mathrm{pu}$ at $t=0.28 \mathrm{~s}$ and then the $q$-component of the reference voltage is suddenly changed from $0.5145 \mathrm{pu}$ to $0.1715 \mathrm{pu}$ at $t=0.743 \mathrm{~s}$. The experimental and simulation results of the islanded microgrid system due to these step changes in the load reference signals are shown in Fig. 4. The results show that the proposed controller can regulate the load voltages within good tracking performance. Moreover, Fig. 4 demonstrates that the simulation results are consistent with experimental data. However, in the experimental 
(a)

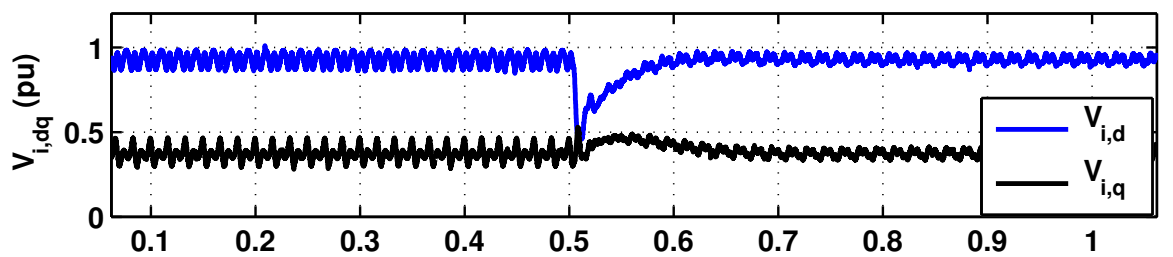

(b)

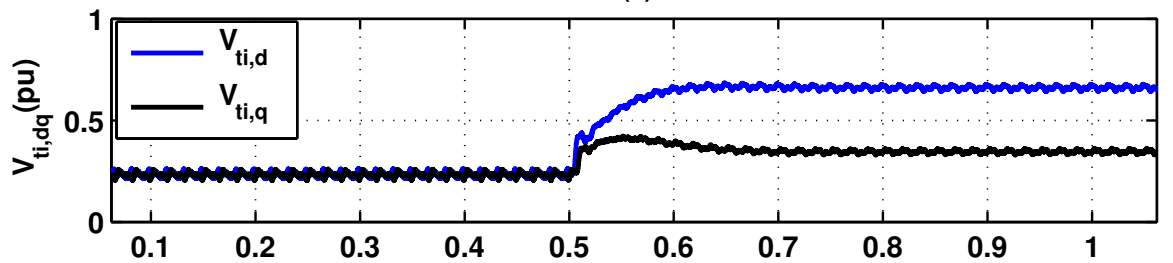

(c)

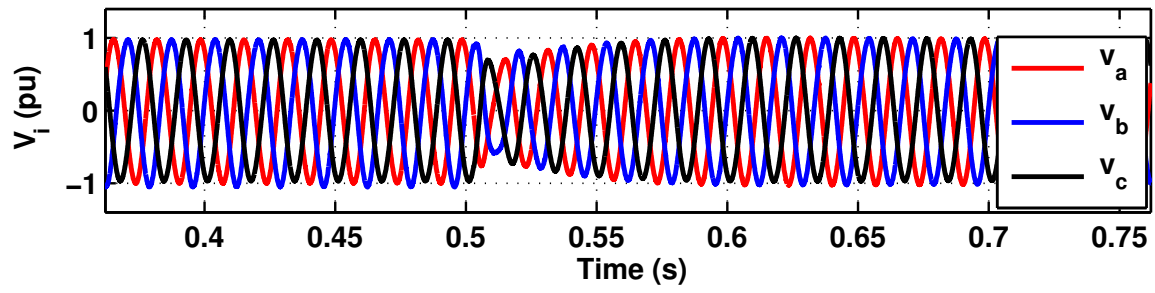

Figure 6: Dynamic responses of the experimental test system due to an inductive load change (a) $d q$-components of the load voltage, (b) control inputs, and (c) instantaneous load voltages

results some ripples in the load voltages are observed due to switching harmonics of the PWM-based voltage-source converter. The amount of ripples is acceptable according to IEEE standards [45].

In the second test, the proposed voltage controller regulates the $d$ and $q$ components of the load voltages at $0.8 p u$ and $0.6 p u$, respectively. The load inductance and load capacitance are also fixed at their nominal values, as given in Table 1. The load resistances in the three phases are equally stepped down from 5 lamps to no lamp ( $\Delta$ configuration) at about $t=200 \mathrm{~ms}$. Fig. 5 shows the dynamical response of the test system due to this resistive load change.

In the third test, the $d$ and $q$ components of the load voltages are set at $0.93 p u$ and $0.37 p u$, respectively. While the load resistances and the load capacitances are fixed at their nominal values, the load inductances in the 
(a)

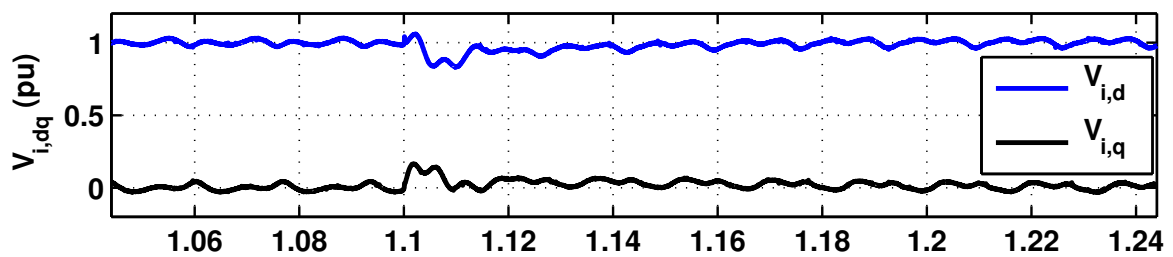

(b)

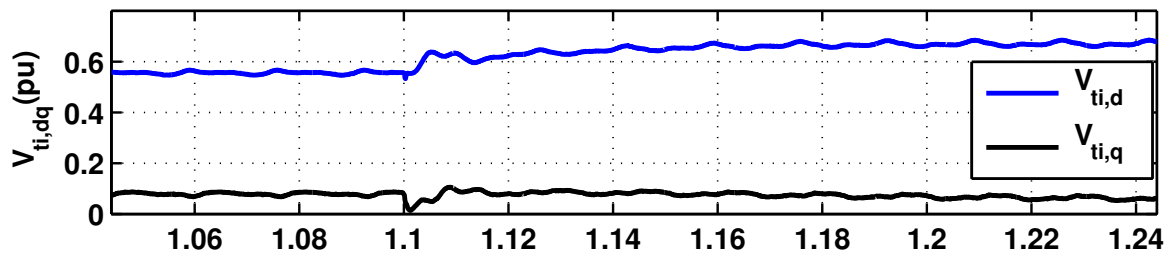

(c)

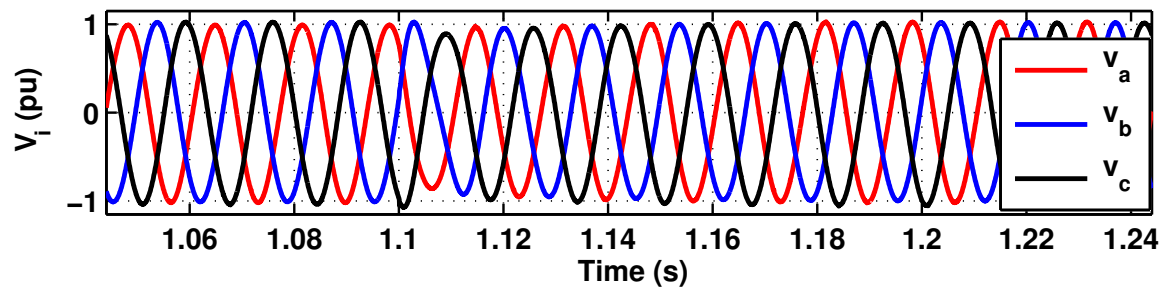

Figure 7: Dynamic responses of the experimental test system due to a capacitive load change (a) $d q$-components of the load voltage, (b) control inputs, and (c) instantaneous load voltages

three phases are suddenly stepped up from $5 \mathrm{mH}$ to $25 \mathrm{mH}$. Then, they suddenly decrease to $5 \mathrm{mH}$ at about $t=0.5 \mathrm{~s}$. Due to the space limitation, the dynamic response of the system for only the second load inductance change is shown in Fig. 6.

In the last test, a change in the load capacitance is considered. To this end, the load capacitances in the three-phases are suddenly changed from the nominal value $850 \mu F$ to $1700 \mu F$ at about $t=1.1 s$, while the load resistances and the load inductances are set based on the values given in Table 1. The dynamic response of the test system is depicted in Fig. 7.

Fig. 5 and Fig. 6 demonstrate that in spite of the large variations in the load resistance and the load inductance, the controller successfully regulates the load voltage with small transients in the responses. Fig. 7 also indicates that the controller adjusts the load voltages within about two 
Table 2: Parameters of the islanded microgrid system with three DG units

\begin{tabular}{|c|c|}
\hline Parameters of RL filter 1 & $R_{t 1}=1 \mathrm{~m} \Omega, \quad L_{t 1}=137.271 \mu \mathrm{H}$ \\
Parameters of RL filter 2 & $R_{t 2}=1.4 m \Omega, \quad L_{t 2}=183.028 \mu \mathrm{H}$ \\
Parameters of RL filter 3 & $R_{t 3}=2.1 m \Omega, \quad L_{t 3}=274.542 \mu \mathrm{H}$ \\
\hline DC bus voltages & $V_{d c}=1500 \mathrm{~V}$ \\
VSC terminal voltage (line-line) & $V_{V S C}=600 \mathrm{KV}$ \\
\hline Transformer parameters & $X_{T}=8 \%$ \\
Transformer voltage ratio & $k=0.6 / 13.8 \mathrm{KV}(\Delta / \mathrm{Y})$ \\
\hline Parameters of RLC load 1 & $R_{1}=350 \Omega, \quad C_{t 1}=60 \mu \mathrm{F}$ \\
(nominal values) & $L_{1}=0.11 \mathrm{H}, \quad R_{l_{1}}=2 \Omega$ \\
Parameters of RLC load 2 & $R_{2}=375 \Omega, \quad C_{t 2}=65 \mu \mathrm{F}$ \\
(nominal values) & $L_{2}=0.1 \mathrm{mH}, \quad R_{l_{2}}=2 \Omega$ \\
Parameters of RLC load 3 & $R_{3}=400 \Omega, \quad C_{t 3}=55 \mu \mathrm{F}$ \\
(nominal values) & $L_{3}=0.12 \mathrm{mH}, \quad R_{l_{3}}=2 \Omega$ \\
\hline System nominal frequency & $f_{0}=60 \mathrm{~Hz}$ \\
\hline Parameters of line 1 & $R_{12}=3.35 \Omega, \quad L_{12}=2.97 \mathrm{mH}$ \\
Parameters of line 2 & $R_{23}=5.025 \Omega, \quad L_{23}=4.5 \mathrm{mH}$ \\
\hline
\end{tabular}

cycles. Therefore, the obtained results confirm that the controller is robust with respect to the load parameter uncertainties. In addition, the coupling between the output signals is small.

The experimental results show that the proposed voltage controller provides satisfactory dynamic performance in terms of voltage tracking and robustness to load parameter variations according to IEEE standards [45].

Remark: It should be noted that the load parameters are uncertain and they do not have step changes in practice. However, the step variations in these parameters lead to the worst-case transient response of the system. Therefore, it can be a good index for the evaluation of the robustness of the designed controller to the load parameter uncertainties.

Scenario 2: In the second scenario, an islanded microgrid consisting of three DG units with the voltage rating of $0.6 \mathrm{kV}$ and power ratings of 1.6 MVA, 1.2 MVA, and 0.8 MVA is considered. The values and the definition of the parameters are provided in Table 2 .

It is assumed that the load resistances $R_{i}$ and inductances $L_{i}, i=1,2,3$ are uncertain up to $\pm 20 \%$ of their nominal values given in Table 2 . Therefore, the uncertainty in this system is in the form of a polytope built by $q=2^{6}$ vertices. 


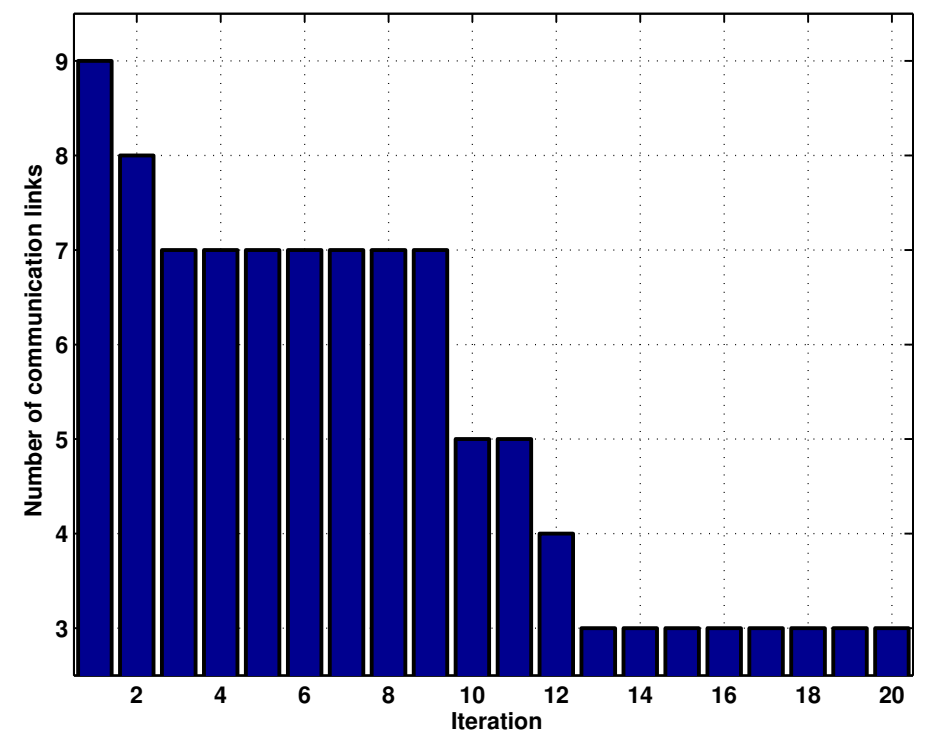

Figure 8: Number of communication link in the feedback controller versus the iteration number

The proposed fixed-order $H_{\infty}$ control method with minimum communication links is used to design a controller for the islanded microgrid consisting of 3 DG units. The final controller is resulted from the following hierarchy of issues:

- Initial centralized controllers are designed by FDRC (Frequency-Domain Robust Controller) Toolbox [40] for each vertex of the polytope.

- To ensure the integral action of the controller, the controller must have six poles at zero. Therefore, one can simply consider six columns/rows of matrix $A_{c}$ to be identically equal to zero (one integrator for each output loop).

- The feedback term of the controller is first designed such that the closed-loop system is robustly stable and its spectral abscissa is minimized.

- The feedforward term of the controller is then designed such that $\left\|W_{s} S(\lambda)\right\|_{\infty}$ is minimized. 
- The parameters of the algorithm given in 3.5 are set as follows: $\epsilon=$ $1 e-10$ and $\alpha=1$.

- The small parameter $\epsilon>0$ in (33) is used to ensure that the weights are well-defined when $\left\|k_{i j}\right\|_{1}^{[h]}=0$. In this case, for almost zero-valued $k_{i j}^{[h]}$, a very high weight is assigned.

- The scalar $\alpha \geq 0$ in (32) characterizes an emphasis on the sparsity of the controller. A larger $\alpha$ leads to a sparser controller whereas $\alpha=0$ renders a centralized fixed-order $H_{\infty}$ controller.

- LMI-based optimization problems are solved using YALMIP [46] as the interface and MOSEK ${ }^{2}$ as the solver.

After 20 iterations, some control structures are obtained. Fig. 8 shows the number of communication links versus the iteration numbers. The resulting decentralized controller in the $20^{\text {th }}$ iteration is given as follows:

$$
\begin{aligned}
& A_{c}=\operatorname{diag}\left(\left[\begin{array}{cccc}
0 & 9.08 e 2 & 0 & -1.07 e 2 \\
0 & -1.885 e 4 & 0 & 1.65 e 3 \\
0 & 1.068 e 2 & 0 & 9.079 e 2 \\
0 & -1.65 e 3 & 0 & -1.885 e 4
\end{array}\right],\left[\begin{array}{cccc}
0 & 2.787 e 2 & 0 & -1.122 e 1 \\
0 & -4.53 e 3 & 0 & 1.096 e 3 \\
0 & 8.99 e 0 & 0 & 2.787 e 2 \\
0 & -1.064 e 3 & 0 & -4.526 e 3
\end{array}\right]\right. \\
& \left.\left[\begin{array}{cccc}
0 & 9.74 e 2 & 0 & -1.17 e 2 \\
0 & -1.84 e 4 & 0 & 1.012 e 3 \\
0 & 1.167 e 2 & 0 & 9.74 e 2 \\
0 & -1.0117 e 3 & 0 & -1.839 e 4
\end{array}\right]\right) \\
& B_{c_{1}}=\operatorname{diag}\left(\left[\begin{array}{cc}
3.24 e 2 & 3.23 e 1 \\
-3.217 e 3 & -3.6157 e 3 \\
-3.0583 e 1 & 3.227 e 2 \\
3.618 e 3 & -3.158 e 3
\end{array}\right],\left[\begin{array}{cc}
-1.518 e 2 & 7.034 e 2 \\
5.711 e 3 & -1.1076 e 4 \\
-7.0023 e 2 & -1.577 e 2 \\
1.101 e 4 & 5.8155 e 3
\end{array}\right],\left[\begin{array}{cc}
3.066 e 2 & 1.8206 e 2 \\
1.206 e 3 & -5.15 e 3 \\
-1.832 e 2 & 3.052 e 2 \\
5.16 e 3 & 1.2445 e 3
\end{array}\right]\right) \\
& C_{c}=\operatorname{diag}\left(\left[\begin{array}{cc}
4.9264 & 2.58 \\
2.2890 & 3.926 \\
-2.5786 & 4.926 \\
-3.9323 & 2.287
\end{array}\right]^{T},\left[\begin{array}{cc}
8.0376 & 3.368 \\
-5.73 & 6.8521 \\
-3.3725 & 8.0425 \\
-6.82 & -5.776
\end{array}\right]^{T},\left[\begin{array}{cc}
5.532 & 4.6948 \\
-3.081 & 8.642 \\
-4.6955 & 5.516 \\
-8.6424 & -3.0794
\end{array}\right]^{T}\right) \\
& D_{c_{1}}=\operatorname{diag}\left(\left[\begin{array}{cc}
6.8763 & -9.899 e-1 \\
1.0153 & 6.871
\end{array}\right],\left[\begin{array}{cc}
2.9976 e 1 & -2.243 \\
2.3468 & 3.0213 e 1
\end{array}\right],\left[\begin{array}{cc}
1.728 e 1 & -1.156 \\
1.163 & 1.7314 e 1
\end{array}\right]\right)
\end{aligned}
$$

\footnotetext{
${ }^{2}$ Available online in http://www.mosek.com
} 
(a)

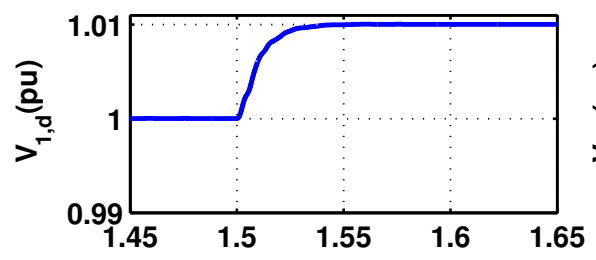

(c)

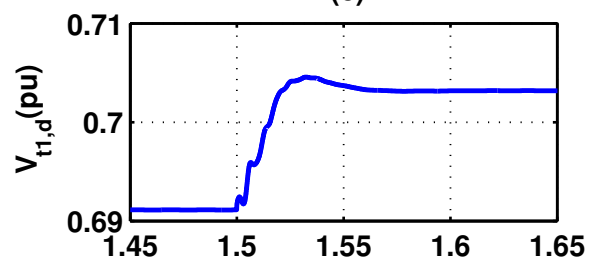

(b)

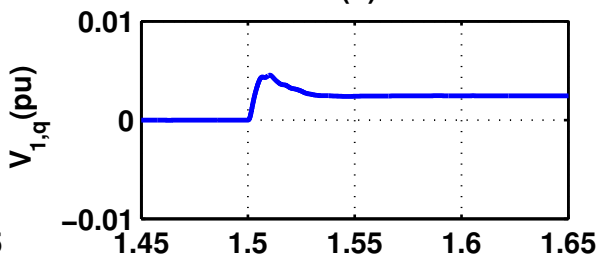

(d)

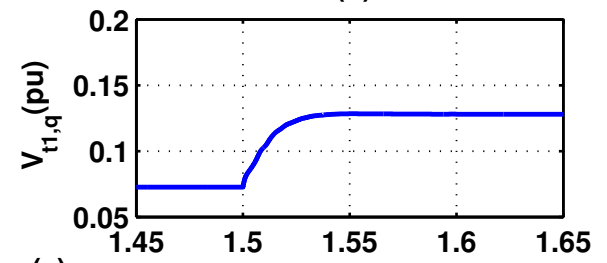

(e)

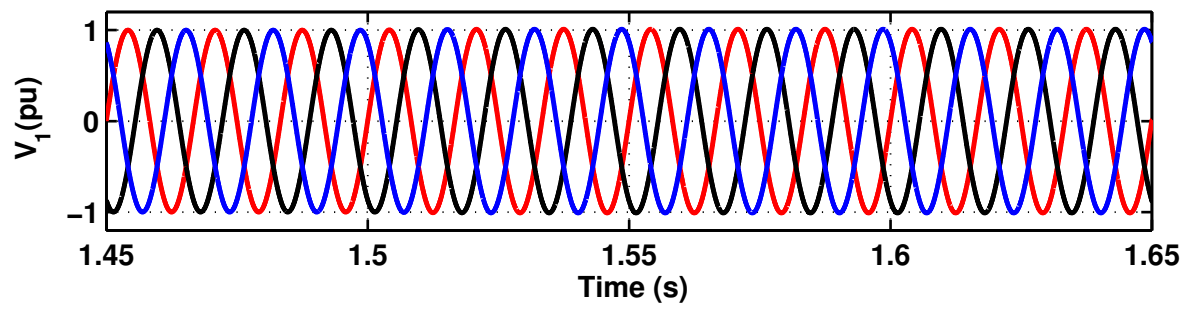

Figure 9: Reference set-point tracking: (a) $d$-component of the load voltage at PCC 1, (b) q-component of the load voltage at PCC 1, (c) $d$-component of the control signal of DG unit 1, (d) q-component of the control signal of DG unit 1, and (e) instantaneous load voltages at PCC 1

The above controller provides $\beta=-4.9219$. The decentralized feedforward term of the controller is resulted after 2 iterations:

$$
\begin{aligned}
& B_{c_{2}}=\operatorname{diag}\left(\left[\begin{array}{cc}
162.22 & -138.43 \\
27.298 & 15.629 \\
140.24 & 163.68 \\
-15.459 & 27.16
\end{array}\right],\left[\begin{array}{cc}
315.49 & 117.7 \\
-27.581 & 60.459 \\
-117.98 & 317.01 \\
-59.828 & -28.427
\end{array}\right],\left[\begin{array}{cc}
351.9 & -96.812 \\
18.278 & 19.767 \\
96.12 & 352.65 \\
-19.944 & 18.106
\end{array}\right]\right) \\
& D_{c_{2}}=\operatorname{diag}\left(\left[\begin{array}{cc}
0.085 & -0.014 \\
0.013 & 0.086
\end{array}\right],\left[\begin{array}{cc}
0.010 & -0.336 \\
0.335 & 0.007
\end{array}\right],\left[\begin{array}{cc}
0.367 & -0.009 \\
0.008 & 0.369
\end{array}\right]\right)
\end{aligned}
$$

The 2DOF feedback-feedforward controller guarantees the robust stability as well as the performance criterion $\left\|W_{s} S(\lambda)\right\|_{\infty}<1.547$ for the whole polytope. 
(a)

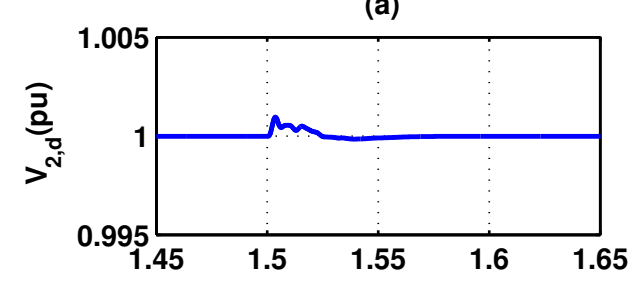

(c)

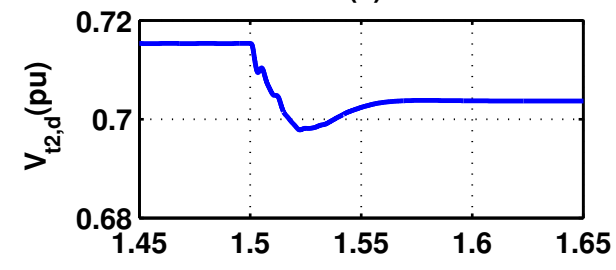

(e)

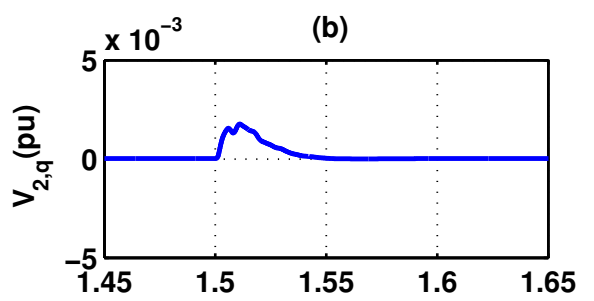

(d)

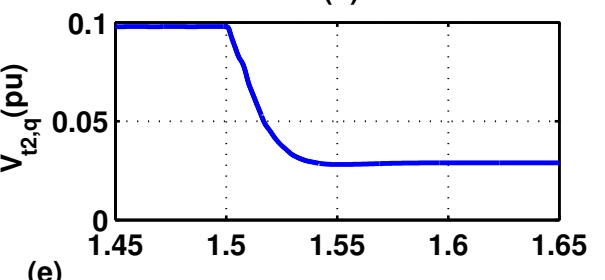

(e)

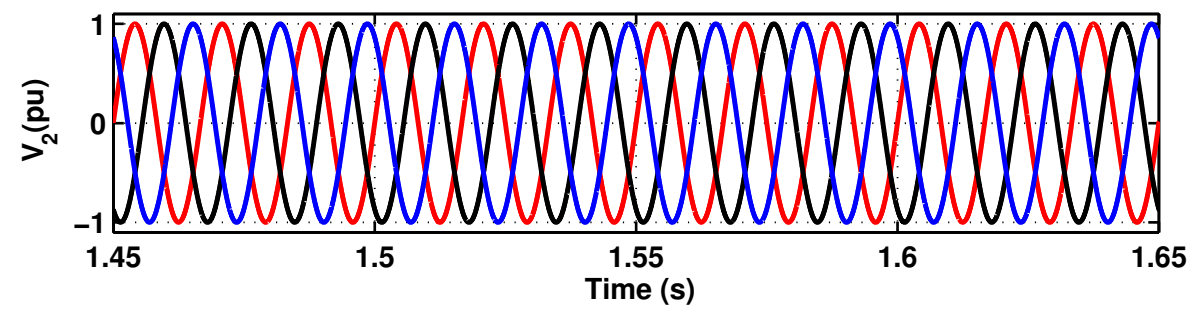

Figure 10: Reference set-point tracking: (a) $d$-component of the load voltage at PCC 2, (b) $q$-component of the load voltage at PCC 2, (c) $d$-component of the control signal of DG unit 2, (d) q-component of the control signal of DG unit 2, and (e) instantaneous load voltages at PCC 2

To evaluate the performance of the designed controller, we consider the capability of the nominal system in voltage setpoint tracking of each DG units. We assume that the load voltages at PCCs are initially regulated at $1 \angle 0^{\circ}$. Then, the output power of DG unit 2 varies due to a change in its local load. Since all three DG units contribute to compensate the total power demand, the PMS determines the following new setpoints for each DG unit at $t=1.5 \mathrm{~s}: V_{1, d q_{r e f}}=1.01 \angle 0.14^{\circ}, V_{2, d q_{r e f}}=1 \angle 0^{\circ}$, and $V_{3, d q_{r e f}}=1 \angle-0.06^{\circ}$. Fig. 9, Fig. 10, and Fig. 11 show the transient response of each DG unit due to the set-point change. The results demonstrate that the proposed controller provides satisfactory dynamic performance according to IEEE standards [45].

Remark: Two-stage 2DOF control design restricts the achievable performance compared to a simultaneous design [27]. However, due to the size 
(a)

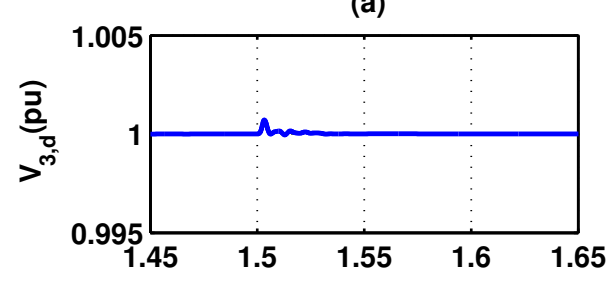

(c)

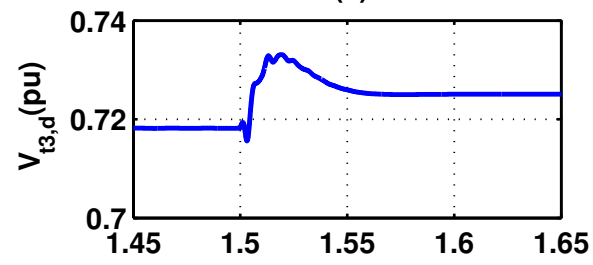

(e)

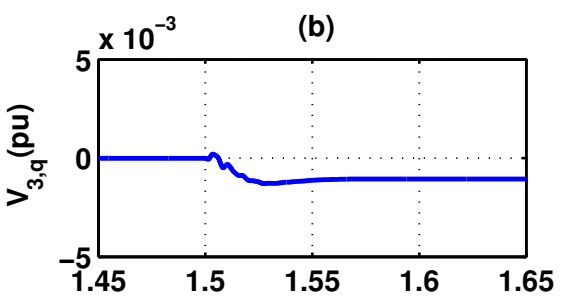

(d)
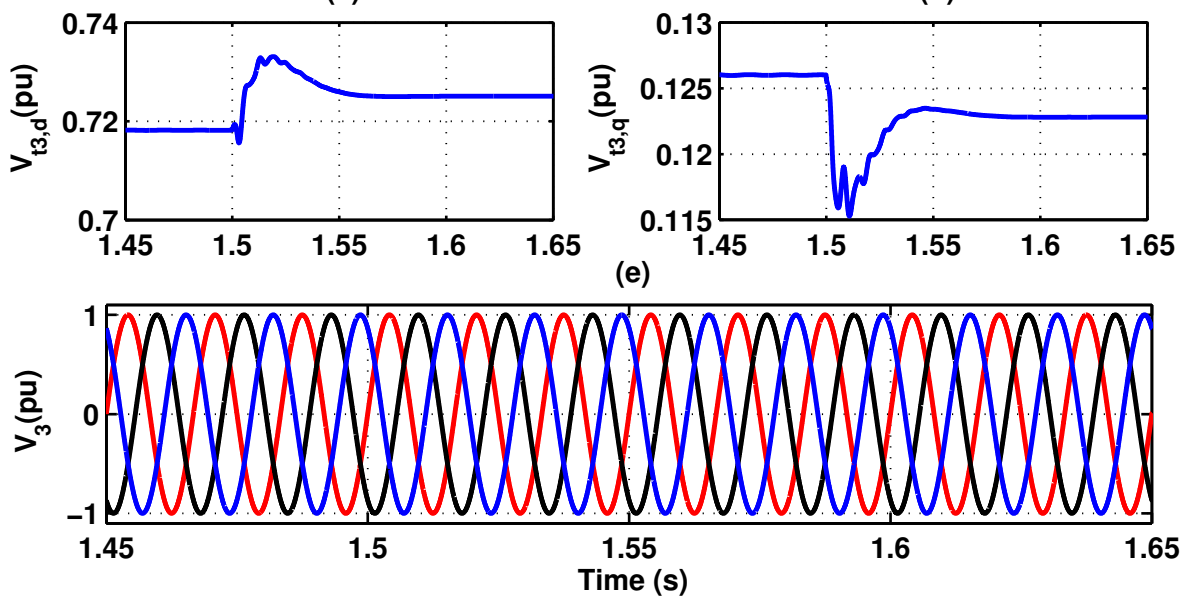

Figure 11: Reference set-point tracking: (a) $d$-component of the load voltage at PCC 3, (b) $q$-component of the load voltage at PCC $3, d$-component of the control signal of DG unit 3, (d) q-component of the control signal of DG unit 3, and (e) instantaneous load voltages at $\mathrm{PCC} 3$

of system and number of vertices in the microgrid case study, SDP solvers encounter numerical problems in the design of one-stage $2 \mathrm{DOF} H_{\infty}$ control through Theorem 2. Therefore, the $2 \mathrm{DOF}$ decentralized voltage controller for the microgrid system is designed in two steps.

\section{CONCLUSION}

This paper proposes a fixed-order decentralized/distributed control strategy for the islanded operation of inverter-interfaced microgrids subject to parameter uncertainties. The uncertainties are imposed by the loads whose parameters can be uncertain. However, it is assumed that the uncertain parameters belong to some prespecified intervals. The controller is designed 
through a solution of a convex optimization problem which relies on a convex relaxation of the cardinality operator and a convex set of fixed-order controllers. The main property of the proposed controller design strategy is that the control structure as well as the control parameters are simultaneously and iteratively designed. To validate the performance of the designed controller, several simulation case studies and experiments are carried out using MATLAB and OPAR-RT Hardware-In-the-Loop, respectively. Both simulation studies and experiments validate the effectiveness of the designed controller in terms of voltage tracking and robustness to the load parameter variations.

\section{ACKNOWLEDGEMENT}

This research work is financially supported by the Swiss National Science Foundation (SNSF) under Grant No. 200020-130528.

\section{REFERENCES}

[1] D. E. Olivares, A. Mehrizi-Sani, A. H. Etemadi, C. A. Canizares, R. Iravani, M. Kazerani, A. H. Hajimiragha, O. Gomis-Bellmunt, M. Saeedifard, R. Palma-Behnke, G. A. Jimenez-Estevez, and N. D. Hatziargyriou, "Trends in microgrid control," IEEE Trans. on Smart Grid, vol. 5, no. 4, pp. 1905 - 1919, July 2014.

[2] J. M. Guerrero, M. Chandorkar, T. Lee, and P. C. Loh, "Advanced control architecture for intelligent microgrids-Part I: Decentralized and hierarchical control," IEEE Trans. on Industrial Electronics, vol. 60, no. 4, pp. 1254-1262, April 2013.

[3] M. Yazdanian and A. Mehrizi-Sani, "Distributed control techniques in microgrids," IEEE Trans. on Smart Grids, vol. 5, no. 6, pp. 2901-2909, November 2014.

[4] A. Bidram, F. L. Lewis, and A. Davoudi, "Distributed control systems for small-scale power networks: using multi agent cooperative control theory," IEEE Control Systems Magazine, vol. 34, no. 6, pp. 56-77, December 2014.

[5] P. Piagi and R. H. Lasseter, "Autonomous control of microgrids," in IEEE power and energy society general meeting, 2006, pp. 1-8. 
[6] J. Lopes, C. Moreira, and A. Madureira, "Defining control strategies for microgrids islanded operation," IEEE Trans. on Power Systems, vol. 21, no. 2, pp. 916-924, May 2006.

[7] J. C. Vasquez, J. M. Guerrero, A. Luna, P. Rodriguez, and R. Teodorescu, "Adaptive droop control applied to voltage-source inverters operating in grid-connected and islanded modes," IEEE Trans. on Industrial Electronics, vol. 56, no. 10, pp. 4088-4096, 2009.

[8] J. C. Vasquez, R. A. Mastromauro, J. M. Guerrero, and M. Liserre, "Voltage support provided by a droop-controlled multifunctional inverter," IEEE Trans. on Industrial Electronics, vol. 56, no. 11, pp. 45104519, 2009.

[9] R. Majumder, G. Ledwich, A. Ghosh, S. Chakrabarti, and F. Zare, "Droop control of converter-interfaced micro source in rural distributed generation," IEEE Trans. on Power Delivery, vol. 25, no. 4, pp. 2768$2778,2010$.

[10] J. M. Guerrero, J. C. Vasquez, J. Matas, L. G. de Vicuna, and M. Castilla, "Hierarchical control of droop-controlled AC and DC microgrids-A general approach towards standardization," IEEE Trans. on Industrial Electronics, vol. 58, no. 1, pp. 158-172, January 2011.

[11] J. Guerrero, P. C. Loh, T. Lee, and M. Chandorkar, "Advanced control architecture for intelligent microgrids-Part II: Power quality, energy storage, and AC/DC microgrids," IEEE Trans. on Industrial Electronics, vol. 60, no. 4, pp. 1263-1275, 2013.

[12] J. M. Guerrero, J. C. Vasquez, J. Matas, M. Castilla, and I. G. de Vicuna, "Control strategy for flexible microgrid based on parallel line-interactive UPS systems," IEEE Trans. on Industrial Electronics, vol. 56, no. 3, pp. 726-736, 2009.

[13] C.-T. Lee, C.-C. Chu, and P.-T. Cheng, "A new droop control method for the autonomous operation of distributed energy resource interface converters," IEEE Trans. on Power Electronics, vol. 28, no. 4, pp. 19801993, April 2013. 
[14] M. Savaghebi, A. Jalilian, J. C. Vasquez, and J. M. Guerrero, "Autonomous voltage unbalance compensation in an islanded droopcontrolled microgrid," IEEE Trans. on Industrial Electronics, vol. 60, no. 4, pp. 1390-1402, April 2013.

[15] P. Kundur, N. J. Balu, and M. G. Lauby, Power System Stability and Control. McGraw-Hill Professional, 1994.

[16] M. C. Chandorkar, D. M. Divan, and R. Adapa, "Control of parallel connected inverters in standalone AC supply systems," IEEE Trans. on Industry Applications, vol. 29, no. 1, pp. 136-143, 1993.

[17] J. Schiffer, R. Ortega, A. Astolfi, J. Raisch, and T. Sezi, "Conditions for stability of droop-controlled inverter-based microgrids," Automatica, vol. 50, no. 10, pp. 2457-2469, October 2014.

[18] N. Pogaku, M. Prodanovic, and T. C. Green, "Modeling, analysis and testing of autonomous operation of an inverter-based microgrid," IEEE Trans. on Power Electronics, vol. 22, no. 2, pp. 613-625, 2007.

[19] H. Karimi, H. Nikkhajoei, and R. Iravani, "Control of an electronicallycoupled distributed resource unit subsequent to an islanding event," IEEE Trans. on Power Delivery, vol. 23, no. 1, pp. 493-501, 2008.

[20] H. Karimi, E. J. Davison, and R. Iravani, "Multivariable servomechanism controller for autonomous operation of a distributed generation unit: Design and performance evaluation," IEEE Trans. on Power Systems, vol. 25, no. 2, pp. 853-865, 2010.

[21] R. Moradi, H. Karimi, and M. Karimi-Ghartemani, "Robust decentralized control for islanded operation of two radially connected DG systems," in IEEE International Symposium on Industrial Electronics (ISIE), Bari, Italy, 2010, pp. 2272-2277.

[22] H. Karimi, A. Yazdani, and R. Iravani, "Robust control of an autonomous four-wire electronically-coupled distributed generation unit," IEEE Trans. on Power Delivery, vol. 26, no. 1, pp. 455-466, January 2011. 
[23] A. H. Etemadi, E. J. Davison, and R. Iravani, "A decentralized robust control strategy for multi-DER microgrid-Part I: Fundamental concepts," IEEE Trans. on Power Delivery, vol. 27, no. 4, pp. 1843-1853, 2012 .

[24] B. Bahrani, M. Saeedifard, A. Karimi, and A. Rufer, "A multivariable design methodology for voltage control of a single-DG-unit microgrid," IEEE Trans. on Industrial Informatics, vol. 9, no. 2, pp. 589 - 599, 2013.

[25] M. Babazadeh and H. Karimi, "A robust two-degree-of-freedom control strategy for an islanded microgrid," IEEE Trans. on Power Delivery, vol. 28, no. 3, pp. 1339-1347, 2013.

[26] A. H. Etemadi, E. J. Davison, and R. Iravani, "A generalized decentralized robust control of islanded microgrids," IEEE Trans. on Power Systems, no. 99, pp. 1-12, 2014.

[27] S. Skogestad and I. Postlethwaite, Multivariable Feedback ControlAnalysis and design. Wiley, 2005.

[28] S. Schuler, M. Gruhler, U. Münz, and F. Allgöwer, "Design of structured static output feedback controllers," in 18th IFAC World Congress, Milano, Italy, 2011.

[29] M. Fardad, F. Lin, and M. R. Jovanovic, "Sparsity-promoting optimal control for a class of distributed systems," in IEEE American Control Conference, San Francisco, CA, USA, 2011.

[30] F. Lin, M. Fardad, and M. R. Jovanovic, "Design of optimal sparse feedback gains via the alternating direction method of multipliers," IEEE Trans. on Automatic Control, vol. 58, no. 9, pp. 2426-2431, 2013.

[31] S. Schuler, U. Münz, and F. Allgöwer, "Decentralized state feedback control for interconnected process systems," in 8th IFAC Symposium on Advanced Control of Chemical Processes, Furama Riverfront, Singapore, 2012.

[32] S. Schuler, P. Li, J. Lam, and F. Allgöwer, "Design of structured dynamic output-feedback controllers for interconnected systems," International Journal of Control, vol. 84, no. 12, pp. 2081-2091, 2011. 
[33] E. J. Candes, M. B. Wakin, and S. P. Boyd, "Enhancing sparsity by reweighted $\ell_{1}$ minimization," Journal of Fourier Analysis and Applications, vol. 14, pp. 877-905, 2008.

[34] M. Fazel, "Matrix rank minimization with applications," Ph.D. dissertation, Department of Electrical Engineering, Stanford University, Stanford, USA, 2002.

[35] E. Beran, "Methods for optimization-based fixed-order control design," Ph.D. dissertation, Technical University of Denmark, Denmark, 1997.

[36] T. Iwasaki, "The dual iteration for fixed-order control," IEEE Trans. on Automatic Control, vol. 44, no. 4, pp. 783-788, April 1999.

[37] P. Apkarian and D. Noll, "Nonsmooth $H_{\infty}$ synthesis," IEEE Trans. on Automatic Control, vol. 51, no. 1, pp. 71-86, 2006.

[38] S. Gumussoy and M. L. Overton, "Fixed-order $H_{\infty}$ controller design via HIFOO, a specialized nonsmooth optimization package," in IEEE American Control Conference, Seattle, USA, 2008, pp. 2750-2754.

[39] A. Karimi and G. Galdos, "Fixed-order $H_{\infty}$ controller design for nonparametric models by convex optimization," Automatica, vol. 46, no. 8, pp. 1388-1394, 2010.

[40] A. Karimi, "Frequency-domain robust controller design: A toolbox for MATLAB," available online: http://la.epfl.ch/FDRC-Toolbox, Automatic Control Laboratory, EPFL, Switzerland, 2012.

[41] S. Boyd, L. E. Ghaoui, E. Feron, and V. Balakrishnan, Linear Matrix Inequalities in System and Control Theory. Philadelphia: SIAM, 1994.

[42] F. Katiraei and M. R. Iravani, "Power management strategies for a microgrid with multiple distributed generation units," IEEE Trans. on Power Systems, vol. 21, no. 4, pp. 1821-1831, 2006.

[43] F. Katiraei, M. R. Iravani, N. Hatziargyriou, and A. Dimeas, "Microgrids management," IEEE Power \&f Energy Magazine, vol. 6, no. 3, pp. 54-65, 2008.

[44] K. Zhou and J. C. Doyle, Essentials of robust control. N.Y.: PrenticeHall, 1998. 
[45] "IEEE recommended practice for monitoring electric power quality, IEEE standard 1159," 2009.

[46] J. Löfberg, "YALMIP: A toolbox for modeling and optimization in MATLAB," in CACSD Conference, 2004. [Online]. Available: http://control.ee.ethz.ch/ joloef/yalmip.php

\section{APPENDICES}

Appendix A: Proof of Theorem 2

Proof. Convex combination of (27) for all vertices leads to the following inequality:

$$
\left[\begin{array}{cccc}
M^{T} P(\lambda)+P(\lambda) M & \star & \star & \star \\
P(\lambda)-M+T^{-1} A(\lambda) T & -2 I & \star & \star \\
0 & \left(T^{-1} B(\lambda)\right)^{T} & -I & \star \\
C(\lambda) T & 0 & D(\lambda) & -\mu I
\end{array}\right]<0
$$

where $\lambda \in \Lambda, P(\lambda)=\sum_{l=1}^{q} \lambda_{l} P^{l}$, and the matrices $(A(\lambda), B(\lambda), C(\lambda), D(\lambda))$ are given in (15). Then, the multiplication of the above inequality on the right by $U_{1}$

$$
U_{1}=\left[\begin{array}{ccc}
I & 0 & 0 \\
P(\lambda) & 0 & 0 \\
0 & I & 0 \\
0 & 0 & I
\end{array}\right]
$$

and on the left by $U_{1}^{T}$ leads to the following inequality:

$$
\left[\begin{array}{ccc}
P(\lambda)\left(T^{-1} A(\lambda) T\right)+\left(T^{-1} A(\lambda) T\right)^{T} P(\lambda) & \star & \star \\
\left(T^{-1} B(\lambda)\right)^{T} P(\lambda) & -I & \star \\
C(\lambda) T & D(\lambda) & -\mu I
\end{array}\right]<0
$$

According to bounded real lemma [41], the above inequality indicates that $\left\|H_{z w}(\lambda)\right\|_{\infty}^{2}<\mu$.

Appendix B: Proof of Lemma 1

Proof. The inequalities of (28) are obtained by the multiplication of (27) on the left by $U_{2}$ and on the right by $U_{2}^{T}$.

$$
U_{2}=\left[\begin{array}{cccc}
T^{-T} & -T^{-T} M^{T}+A^{l^{T}} T^{-T} & 0 & 0 \\
0 & T^{-T} & 0 & 0 \\
0 & 0 & I & 0 \\
0 & 0 & 0 & I
\end{array}\right]
$$

